\title{
Semidefinite descriptions of the convex hull of rotation matrices
}

\author{
James Saunderson Pablo A. Parrilo Alan S. Willsky *
}

March 20, 2014

\begin{abstract}
We study the convex hull of $S O(n)$, thought of as the set of $n \times n$ orthogonal matrices with unit determinant, from the point of view of semidefinite programming. We show that the convex hull of $S O(n)$ is doubly spectrahedral, i.e. both it and its polar have a description as the intersection of a cone of positive semidefinite matrices with an affine subspace. Our spectrahedral representations are explicit, and are of minimum size, in the sense that there are no smaller spectrahedral representations of these convex bodies.
\end{abstract}

\section{Introduction}

Optimization problems where the decision variables are constrained to be in the set of orthogonal matrices

$$
O(n):=\left\{X \in \mathbb{R}^{n \times n}: X^{T} X=I\right\}
$$

arise in many contexts (see, e.g., [24, 23] and references therein), particularly when searching over Euclidean isometries or orthonormal frames. In some situations, especially those arising from physical problems, we require the additional constraint that the decision variables be in the set of rotation matrices

$$
S O(n):=\left\{X \in \mathbb{R}^{n \times n}: X^{T} X=I, \operatorname{det}(X)=1\right\}
$$

representing Euclidean isometries that also preserve orientation. For example, these additional constraints arise in problems involving attitude estimation for spacecraft [25] or pose estimation in computer vision applications [17], or in understanding protein folding [21]. The unit determinant constraint is important in these situations because we typically cannot reflect physical objects such as spacecraft or molecules.

The set of $n \times n$ rotation matrices is non-convex, so optimization problems over rotation matrices are ostensibly non-convex optimization problems. An important approach to global non-convex optimization is to approximate the original non-convex problem with a tractable convex optimization problem. In some circumstances it may even be possible to exactly reformulate the original non-convex problem as a tractable convex problem. This approach to global optimization via convexification has been very influential in combinatorial optimization [31], and more generally in polynomial optimization via the machinery of moments and sums of squares [4]. As an example of a problem amenable to this approach, in Section 2 we describe the problem of jointly estimating

${ }^{*}$ The authors are with the Laboratory for Information and Decision Systems, Department of Electrical Engineering and Computer Science, Massachusetts Institute of Technology, Cambridge MA 02139, USA. Email: $\{$ jamess,parrilo,willsky\}@mit.edu. 
the attitude and spin-rate of a spinning satellite and show how to reformulate this ostensibly nonconvex problem as a convex optimization problem that, using the constructions in this paper, can be expressed as a semidefinite program.

When we attempt to convexify optimization problems involving rotation matrices two natural geometric objects arise. The first of these is the convex hull of $S O(n)$ which we denote, throughout, by conv $S O(n)$. The second convex body of interest in this paper is the polar of $S O(n)$, the set of linear functionals that take value at most one on $S O(n)$, i.e.,

$$
S O(n)^{\circ}=\left\{Y \in \mathbb{R}^{n \times n}:\langle Y, X\rangle \leq 1 \text { for all } X \in S O(n)\right\}
$$

where we have identified $\mathbb{R}^{n \times n}$ with its dual space via the trace inner product $\langle Y, X\rangle=\operatorname{tr}\left(Y^{T} X\right)$. These two convex bodies are closely related. Since conv $S O(n)$ is closed and contains the origin it follows from basic results of convex analysis [28, Theorem 14.5] that conv $S O(n)=S O(n)^{\circ \circ}$.

We also study the convex hull and the polar of orthogonal matrices in this paper. It is wellknown that these correspond to commonly used matrix norms (see, e.g., [29]). The convex hull of $O(n)$ is the operator norm ball, the set of $n \times n$ matrices with largest singular value at most one, and the polar of $O(n)$ is the nuclear norm ball, the set of $n \times n$ matrices such that the sum of the singular values is at most one, i.e.

$$
\operatorname{conv} O(n)=\left\{X \in \mathbb{R}^{n \times n}: \sigma_{1}(X) \leq 1\right\} \quad \text { and } \quad O(n)^{\circ}=\left\{X \in \mathbb{R}^{n \times n}: \sum_{i=1}^{n} \sigma_{i}(X) \leq 1\right\} .
$$

Note that $O(n)$ is the (disjoint) union of $S O(n)$ and the set $S O^{-}(n):=\left\{X \in \mathbb{R}^{n \times n}: X^{T} X=\right.$ $I, \operatorname{det}(X)=-1\}$. As such, it follows from basic properties of the polar [28, Corollary 16.5.2] that

$$
O(n)^{\circ}=S O(n)^{\circ} \cap S O^{-}(n)^{\circ}
$$

allowing us to deduce properties of $O(n)^{\circ}$ from those of $S O(n)^{\circ}$. On the other hand we show in Proposition 4.7 that

$$
\operatorname{conv} S O(n)=\operatorname{conv} O(n) \cap(n-2) S O^{-}(n)^{\circ}
$$

allowing us to deduce properties of conv $S O(n)$ from properties of $\operatorname{conv} O(n)$ and $S O^{-}(n)^{\circ}$. Figure 1 illustrates the differences between conv $S O(n)$ and conv $O(n)$ and the relationship described in (3).

The convex bodies conv $S O(n)$ and conv $O(n)$ are examples of orbitopes, a family of highly symmetric convex bodies that arise from representations of groups [29, 3, 2]. Suppose a compact group $G$ acts on $\mathbb{R}^{n}$ by linear transformations and $x_{0} \in \mathbb{R}^{n}$. Then the orbit of $x_{0}$ under $G$ is

$$
G \cdot x_{0}=\left\{g \cdot x_{0}: g \in G\right\} \subset \mathbb{R}^{n}
$$

and the corresponding orbitope is conv $\left(G \cdot x_{0}\right)$, the convex hull of the orbit. The sets $O(n)$ and $S O(n)$ defined above can be thought of as the orbit of the identity matrix $I \in \mathbb{R}^{n \times n}$ under the linear action of the groups $O(n)$ and $S O(n)$, respectively, by right multiplication on $n \times n$ matrices. The corresponding orbitopes are known as the tautological $O(n)$ orbitope and the tautological $S O(n)$ orbitope respectively [29]. The set $S O^{-}(n)$ can be viewed as the orbit of $R:=\operatorname{diag}^{*}(1,1, \ldots, 1,-1)$, the diagonal matrix with diagonal entries $(1,1, \ldots, 1,-1)$, under the same $S O(n)$ action on $n \times n$ matrices. Note that $S O^{-}(n)$ is then the image of $S O(n)$ under the invertible linear map $X \mapsto R \cdot X$. 


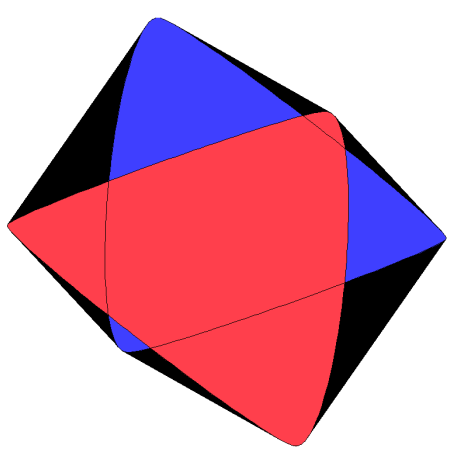

(a) A 2-dimensional projection of conv $S O(3)$ (red), conv $S O^{-}(3)$ (blue), and $\operatorname{conv} O(3)=$ conv $\left[S O(3) \cup S O^{-}(3)\right]$ (black).

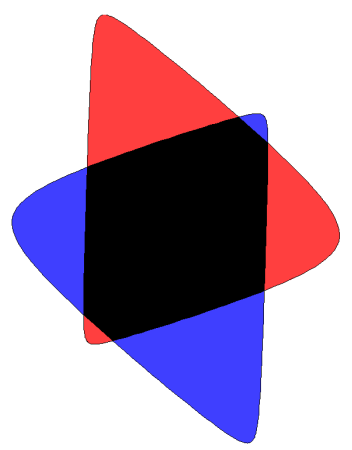

(b) The corresponding 2-dimensional section of $S O(3)^{\circ}$ (red), $S^{-}(3)^{\circ}$ (blue), and $O(3)^{\circ}=$ $S O(3)^{\circ} \cap S O^{-}(3)^{\circ}$ (black).

Figure 1: Pictures of some of the convex bodies considered in this paper. These were created by optimizing 100 linear functionals over each of these sets to obtain 100 boundary points. The optimization was performed by implementing our spectrahedral representations in the parser YALMIP [20], and solving the semidefinite programs numerically using SDPT3 [33].

Spectrahedra For convex reformulations or relaxations involving the convex hull of $S O(n)$ to be useful from a computational point of view we need an effective description of the convex body conv $S O(n)$. One effective way to describe a convex body is to express it as the intersection of the cone of symmetric positive semidefinite matrices with an affine subspace. Such convex bodies are called spectrahedra [26] and are natural generalizations of polyhedra. Algebraically, a convex subset $C$ of $\mathbb{R}^{n}$ (containing the origin in its interior ${ }^{1}$ ) is a spectrahedron if it can be expressed as the feasible region of a linear matrix inequality of the form

$$
C=\left\{x \in \mathbb{R}^{n}: I_{m}+\sum_{i=1}^{n} A_{i} x_{i} \succeq 0\right\}
$$

where $I_{m}$ is the $m \times m$ identity matrix, $A_{1}, A_{2}, \ldots, A_{n}$ are $m \times m$ real symmetric matrices and $M \succeq 0$ means that $M$ is a symmetric positive semidefinite matrix. If the matrices $A_{i}$ are $m \times m$, we call the description (5) a spectrahedral representation of size $m$.

Giving a spectrahedral representation for a convex set has algebraic, geometric, and algorithmic implications. Algebraically, a spectrahedral representation of $C$ of size $m$ as in (5) tells us that the degree $m$ polynomial $p(x)=\operatorname{det}\left(I+\sum_{i=1}^{n} A_{i} x_{i}\right)$ vanishes on the boundary of $C$, and that $C$ itself can be written as the region defined by $m$ polynomial inequalities (i.e. it is a basic closed semi-algebraic set) [27, Theorem 20]. Geometrically, a spectrahedral representation of $C$ gives information about its facial structure. For example, it is known that all faces of a spectrahedron are exposed (i.e., can be obtained as the intersection of the spectrahedron with a supporting hyperplane), since the same is true for the positive semidefinite cone.

From the point of view of optimization, problems involving minimizing a linear functional over a spectrahedron are called semidefinite optimization problems [4] and are natural generalizations

\footnotetext{
${ }^{1}$ We can assume this without loss of generality by translating $C$ and restricting to its affine hull
} 
of the more well-known class of linear programming problems. Semidefinite optimization problems can be solved (to any desired accuracy) in time polynomial in $n$ and $m$.

The convex sets that can be obtained as the images of spectrahedra under linear maps are also of interest. Indeed to minimize a linear functional over a projection of a spectrahedron, one can simply lift the linear functional and minimize it over the spectrahedron itself using methods for semidefinite optimization. We say a convex body has a PSD lift if it has a description as a projection of a spectrahedron (see Section 5.2). PSD lifts are important because they form a strictly larger family of convex sets than spectrahedra, and because some spectrahedra have PSD lifts that are much more concise than their smallest spectrahedral representations (generalizing the notion of extended formulations for polyhedra). On the other hand convex bodies that have PSD lifts do not enjoy the same nice algebraic and geometric properties as spectrahedra -indeed they are semialgebraic but not necessarily basic semialgebraic, and are not necessarily facially exposed [4].

Throughout much of the paper we consider only spectrahedral representations, confining our discussion of PSD lifts to Section 5.2.

Doubly spectrahedral convex sets In this paper we are interested in both $S O(n)^{\circ}$ and conv $S O(n)$, and so study both from the point of view of semidefinite programming. For finite sets $S$, both $S^{\circ}$ and conv $S$ are polyhedra. On the other hand, for infinite sets $S$, usually neither $S^{\circ}$ nor conv $S$ are spectrahedra. Even if a convex set is a spectrahedron, typically its polar is not a spectrahedron (see Section 6). We use the term doubly spectrahedral convex sets to refer to those very special convex sets $C$ with the property that both $C$ and $C^{\circ}$ are spectrahedra.

Main contribution The main contribution of this paper is to establish that conv $S O(n)$ is doubly spectrahedral and to give explicit spectrahedral representations of both $S O(n)^{\circ}$ and conv $S O(n)$.

Main proof technique The main idea behind our representations is that we start with a parameterization of $S O(n)$, rather than working with the defining equations in (2). The parameterization is a direct (and classical) generalization of the widely used unit quaternion parameterization of $S O(3)$. In higher dimensions the unit quaternions are replaced with $\operatorname{Spin}(n)$, a multiplicative subgroup of the invertible elements of a Clifford algebra. In the cases $n=2$ and $n=3$ it is relatively straightforward to produce our semidefinite representations directly from this parameterization. For $n \geq 4$ the parameterization does not immediately yield our semidefinite representations. The additional arguments required to establish the correctness of our representations for $n \geq 4$ form the main technical contribution of the paper.

\subsection{Statement of results}

In this section we explicitly state the spectrahedral representations that we prove are correct in subsequent sections of the paper. In particular we state spectrahedral representations for $S O(n)^{\circ}$ and conv $S O(n)$, as well as a spectrahedral representation of $O(n)^{\circ}$, the nuclear norm ball. All the spectrahedral representations stated in this section are of minimum size (see Theorem 1.4). The reader primarily interested in implementing our semidefinite representations should find all the information necessary to do so in this section.

Matrices of the spectrahedral representations Our main results are stated in terms of a collection of symmetric $2^{n-1} \times 2^{n-1}$ matrices denoted $\left(A_{i j}\right)_{1 \leq i, j \leq n}$. We give concrete descriptions of 
them here in terms of the Kronecker product of $2 \times 2$ matrices, deferring more invariant descriptions to Appendix A. The matrices $A_{i j}$ can be expressed as

$$
A_{i j}=-P_{\text {even }}^{T} \lambda_{i} \rho_{j} P_{\text {even }}
$$

where $\left(\lambda_{i}\right)_{i=1}^{n}$ and $\left(\rho_{i}\right)_{i=1}^{n}$ are the $2^{n} \times 2^{n}$ skew-symmetric matrices defined concretely by

$$
\begin{aligned}
& \lambda_{i}=\overbrace{\left[\begin{array}{cc}
1 & 0 \\
0 & -1
\end{array}\right] \otimes \cdots \otimes\left[\begin{array}{cc}
1 & 0 \\
0 & -1
\end{array}\right]}^{i-1} \otimes\left[\begin{array}{cc}
0 & -1 \\
1 & 0
\end{array}\right] \otimes \overbrace{\left[\begin{array}{cc}
1 & 0 \\
0 & 1
\end{array}\right] \otimes \cdots \otimes\left[\begin{array}{ll}
1 & 0 \\
0 & 1
\end{array}\right]}^{n-i} \\
& \rho_{i}=\underbrace{\left[\begin{array}{cc}
1 & 0 \\
0 & 1
\end{array}\right] \otimes \cdots \otimes\left[\begin{array}{cc}
1 & 0 \\
0 & 1
\end{array}\right]}_{i-1} \otimes\left[\begin{array}{cc}
0 & -1 \\
1 & 0
\end{array}\right] \otimes \underbrace{\left[\begin{array}{cc}
1 & 0 \\
0 & -1
\end{array}\right] \otimes \cdots \otimes\left[\begin{array}{cc}
1 & 0 \\
0 & -1
\end{array}\right]}_{n-i}
\end{aligned}
$$

and $P_{\text {even }}$ is the $2^{n} \times 2^{n-1}$ matrix with orthonormal columns

$$
P_{\text {even }}=\frac{1}{2}\left[\begin{array}{l}
I_{2^{n-1}}+\left[\begin{array}{cc}
1 & 0 \\
0 & -1
\end{array}\right] \otimes \cdots \otimes\left[\begin{array}{cc}
1 & 0 \\
0 & -1
\end{array}\right] \\
I_{2^{n-1}}-\left[\begin{array}{cc}
1 & 0 \\
0 & -1
\end{array}\right] \otimes \cdots \otimes\left[\begin{array}{cc}
1 & 0 \\
0 & -1
\end{array}\right]
\end{array}\right] .
$$

Note that $P_{\text {even }}^{T} M P_{\text {even }}$ just selects a particular $2^{n-1} \times 2^{n-1}$ principal submatrix of $M$. Since, for any pair $1 \leq i, j \leq n, \lambda_{i}$ and $\rho_{j}$ are skew symmetric and commute it follows that each $A_{i j}$ is symmetric. Furthermore since $\lambda_{i}$ and $\rho_{j}$ are signed permutation matrices, so is $-\lambda_{i} \rho_{j}$. From this we can see that all of the entries of the $A_{i j}$ are 0,1 , or -1 .

Spectrahedral representations The following, which we prove in Section 4, is the main technical result of the paper.

Theorem 1.1. The polar of $S O(n)$ is a spectrahedron. Explicitly

$$
S O(n)^{\circ}=\left\{Y \in \mathbb{R}^{n \times n}: \sum_{i, j=1}^{n} A_{i j} Y_{i j} \preceq I_{2^{n-1}}\right\}
$$

where the $2^{n-1} \times 2^{n-1}$ matrices $A_{i j}$ are defined in (6).

Since $O(n)=S O(n) \cup S O^{-}(n)$ as a corollary of Theorem 1.1 we obtain a spectrahedral representation of $O(n)^{\circ}=S O(n)^{\circ} \cap S O^{-}(n)^{\circ}$.

Theorem 1.2. The polar of $O(n)$ is a spectrahedron. Explicitly

$$
O(n)^{\circ}=\left\{Y \in \mathbb{R}^{n \times n}: \sum_{i, j=1}^{n} A_{i j} Y_{i j} \preceq I_{2^{n-1}}, \sum_{i, j=1}^{n} A_{i j}[R Y]_{i j} \preceq I_{2^{n-1}}\right\} .
$$

where $R=\operatorname{diag}^{*}(1,1, \ldots, 1,-1)$. 
Just because a convex set $C$ is a spectrahedron does not, in general, mean that its polar is also spectrahedron (see Section 6 for a simple example). Even if we are in the special case where $C$ is doubly spectrahedral, we cannot simply dualize a spectrahedral representation of $C$ to obtain a spectrahedral representation of its polar. Nevertheless, by a separate argument we can show that conv $S O(n)=\operatorname{conv} O(n) \cap(n-2) S O^{-}(n)^{\circ}$ (Proposition 4.7) to obtain a spectrahedral representation of conv $S O(n)$. We explain how this works in detail in Section 4.3.

Theorem 1.3. The convex hull of $S O(n)$ is a spectrahedron. Explicitly

$$
\operatorname{conv} S O(n)=\left\{X \in \mathbb{R}^{n \times n}:\left[\begin{array}{cc}
0 & X \\
X^{T} & 0
\end{array}\right] \preceq I_{2 n}, \quad \sum_{i, j=1}^{n} A_{i j}[R X]_{i j} \preceq(n-2) I_{2^{n-1}}\right\} .
$$

In the special cases $n=2$ and $n=3$ these representations can be simplified to

$$
\begin{aligned}
& \operatorname{conv} S O(2)=\left\{\left[\begin{array}{cc}
c & -s \\
s & c
\end{array}\right] \in \mathbb{R}^{2 \times 2}:\left[\begin{array}{cc}
1+c & s \\
s & 1-c
\end{array}\right] \succeq 0\right\} \quad \text { and } \\
& \operatorname{conv} S O(3)=\left\{X \in \mathbb{R}^{3 \times 3}: \sum_{i, j=1}^{3} A_{i j}[R X]_{i j} \preceq I_{4}\right\} \\
& =\left\{X \in \mathbb{R}^{3 \times 3}:\left[\begin{array}{cccc}
1-X_{11}-X_{22}+X_{33} & X_{13}+X_{31} & X_{12}-X_{21} & X_{23}+X_{32} \\
X_{13}+X_{31} & 1+X_{11}-X_{22}-X_{33} & X_{23}-X_{32} & X_{12}+X_{21} \\
X_{12}-X_{21} & X_{23}-X_{32} & 1+X_{11}+X_{22}+X_{33} & X_{31}-X_{13} \\
X_{23}+X_{32} & X_{12}+X_{21} & X_{31}-X_{13} & 1-X_{11}+X_{22}-X_{33}
\end{array}\right] \succeq 0\right\} \text {. }
\end{aligned}
$$

We note that the representation of conv $S O(3)$ described in Sanyal et al. [29, Proposition 4.1] can be obtained from the spectrahedral representation for conv $S O(3)$ given here by conjugating by a signed permutation matrix, establishing that the two representations are equivalent.

In Section 5 we prove that our spectrahedral representations in Theorems 1.1, 1.2, 1.3 are of minimum size. We do so by establishing lower bounds on the minimum size of spectrahedral representations of $S O(n)^{\circ}$, conv $S O(n)$ and $O(n)^{\circ}$ that match the upper bounds given by our constructions.

Theorem 1.4. If $n \geq 1$ the minimum size of a spectrahedral representation of $O(n)^{\circ}$ is $2^{n}$. If $n \geq 2$ the minimum size of a spectrahedral representation of $S O(n)^{\circ}$ is $2^{n-1}$. If $n \geq 4$ the minimum size of a spectrahedral representation of conv $S O(n)$ is $2^{n-1}+2 n$. The minimum size of a spectrahedral representation of conv $S O(3)$ is 4 .

Representations as PSD lifts Given a spectrahedral representation of size $m$ of a convex set $C$ (with the origin in its interior), by applying a straightforward conic duality argument (see, for example, [12, Proposition 3.1]) we can obtain a PSD lift of $C^{\circ}$. This representation, however, is usually not a spectrahedral representation.

Example 1.5. Theorems 1.2 and 1.4 tell us that the smallest spectrahedral representation of $O(n)^{\circ}$, the nuclear norm ball, has size $2^{n}$. Yet by dualizing the size $2 n$ spectrahedral representation of conv $O(n)$ (given in Proposition 4.8 to follow) we obtain a PSD lift of $O(n)^{\circ}$ of size $2 n$

$$
O(n)^{\circ}=\left\{Z \in \mathbb{R}^{n \times n}: \exists X, Y \text { s.t. }\left[\begin{array}{cc}
X & Z \\
Z^{T} & Y
\end{array}\right] \succeq 0, \operatorname{tr}(X)+\operatorname{tr}(Y)=2\right\} .
$$

This is equivalent to the representation given by Fazel [9] for the nuclear norm ball.

By dualizing, in a similar fashion, the spectrahedral representation of $S O(n)^{\circ}$ we obtain a representation of conv $S O(n)$ as the projection of a spectrahedron, i.e. a PSD lift of conv $S O(n)$. 
Corollary 1.6. The convex hull of $S O(n)$ can be expressed as a projection of the $2^{n-1} \times 2^{n-1}$ positive semidefinite matrices with trace one as

$$
\operatorname{conv} S O(n)=\left\{\left[\begin{array}{cccc}
\left\langle A_{11}, Z\right\rangle & \left\langle A_{12}, Z\right\rangle & \cdots & \left\langle A_{1 n}, Z\right\rangle \\
\left\langle A_{21}, Z\right\rangle & \left\langle A_{22}, Z\right\rangle & \cdots & \left\langle A_{2 n}, Z\right\rangle \\
\vdots & \vdots & \ddots & \vdots \\
\left\langle A_{n 1}, Z\right\rangle & \left\langle A_{n 2}, Z\right\rangle & \cdots & \left\langle A_{n n}, Z\right\rangle
\end{array}\right]: Z \succeq 0, \operatorname{tr}(Z)=1\right\} .
$$

In some situations it may be preferable to use this representation of conv $S O(n)$ rather than the spectrahedral representation in Theorem 1.3.

\subsection{Related work}

That the convex hull of $O(n)$ is a spectrahedron is a classical result. It was not until recently that Sanyal et al. [29] established that $O(n)^{\circ}$ is a spectrahedron by explicitly giving a (non-optimal) size $\left(\begin{array}{c}2 n \\ n\end{array}\right)$ spectrahedral representation. In the same paper, Sanyal et al. study numerous $S O(n)$ and $O(n)$-orbitopes considering both convex geometric aspects such as their facial structure and Caratheodory number, and algebraic aspects such as their algebraic boundary and whether they are spectrahedra. They describe (previously known) spectrahedral representations of conv $S O(2)$ and conv $S O(3)$. The representation for conv $S O(3)$ given in [29, Eq. 4.1] is equivalent to our representation in Theorem 1.3, and the representation given in [29, Eq. 4.2] is equivalent to

$$
\operatorname{conv} S O(3)=\left\{\left[\begin{array}{ccc}
Z_{11}-Z_{22}-Z_{33}+Z_{44} & -2 Z_{13}-2 Z_{24} & -2 Z_{12}+2 Z_{34} \\
2 Z_{13}-2 Z_{24} & Z_{11}+Z_{22}-Z_{33}-Z_{44} & -2 Z_{14}-2 Z_{23} \\
2 Z_{12}+2 Z_{34} & 2 Z_{14}-2 Z_{23} & Z_{11}-Z_{22}+Z_{33}-Z_{44}
\end{array}\right]: Z \succeq 0, \operatorname{tr}(Z)=1\right\}
$$

which can be obtained by specializing Corollary 1.6. Sanyal et al. raise the general question of whether conv $S O(n)$ is a spectrahedron for all $n$ (which we answer in the affirmative), and more broadly ask for a classification of the $S O(n)$-orbitopes that are spectrahedra.

Earlier work on orbitopes in the context of convex geometry includes the work of Barvinok and Vershik [3] who consider orbitopes of finite groups in the context of combinatorial optimization, Barvinok and Blekherman [2], who used asymptotic volume computations to show that there are many more non-negative polynomials than sums of squares (among other things), and Longinetti et al. [21] who studied $S O(3)$-orbitopes with a view to applications in protein structure determination. More recently Sinn [32] has studied in detail the algebraic boundary of four-dimensional $S O(2)$ orbitopes as well as the Barvinok-Novik orbitopes.

\subsection{Notation}

In this section we gather notation not explicitly defined elsewhere in the paper. We use $\mathcal{S}^{m}$ and $\mathcal{S}_{+}^{m}$ to denote the space of symmetric $m \times m$ matrices and the cone of positive semidefinite matrices respectively. If $\mathcal{U} \subset \mathbb{R}^{n}$ is a subspace then $\pi_{\mathcal{U}}: \mathbb{R}^{n} \rightarrow \mathcal{U}$ is the orthogonal projector onto $\mathcal{U}$ and $\pi_{\mathcal{U}}^{*}: \mathcal{U} \rightarrow \mathbb{R}^{n}$ is its adjoint. If the subspace in question is the subspace of diagonal matrices $\mathcal{D} \subset \mathbb{R}^{n \times n}$ we occasionally also use diag $:=\pi_{\mathcal{D}}$ and diag* $^{*}:=\pi_{\mathcal{D}}^{*}$. We frequently use the matrix $R=\operatorname{diag}^{*}(1,1, \ldots, 1,-1) \in \mathbb{R}^{n \times n}$. It could be replaced, throughout, by any orthogonal self-adjoint matrix with determinant -1 . We use the shorthand $[n]$ for the set $\{1,2, \ldots, n\}$ and $\mathcal{I}_{\text {even }}$ for the set of subsets of $[n]$ with even cardinality.

\subsection{Outline}

The remainder of the paper is organized as follows. In Section 2 we describe a problem in satellite attitude estimation that can be reformulated as a semidefinite program using the ideas in this paper. 


\begin{tabular}{|c|c|c|c|}
\hline$S$ & & $S O(n)$ & $O(n)$ \\
\hline \multirow{6}{*}{$S^{\circ}$} & Definition & $\left\{X \in \mathbb{R}^{n \times n}: X^{T} X=I, \operatorname{det}(X)=1\right\}$ & $\left\{X \in \mathbb{R}^{n \times n}: X^{T} X=I\right\}$ \\
\hline & & $S O(n)^{\circ}$ & $O(n)^{\circ}=$ Nuclear norm ball \\
\hline & Diagonal slice & Polar of parity polytope (Prop. 3.4) & Cross-polytope \\
\hline & Spectrahedral & Size: $2^{n-1}$ & Size: $2^{n} \quad($ Thm 1.2$)$ \\
\hline & representation & Matching lower bound (Thm 1.4) & Matching lower bound (Thm 1.4) \\
\hline & PSD lift & Size: $2^{n-1}$ & Size: $2 n$ \\
\hline \multirow[t]{5}{*}{$\begin{array}{l}S^{\circ \circ}= \\
\operatorname{conv} S\end{array}$} & & $\operatorname{conv} S O(n)$ & conv $O(n)=$ Operator norm ball \\
\hline & Diagonal slice & Parity Polytope & Hypercube \\
\hline & $\begin{array}{l}\text { Spectrahedral } \\
\text { representation }\end{array}$ & Size: $\left\{\begin{array}{ll}2^{n-1}+2 n & n \geq 4 \\
4 & n=3\end{array}(\right.$ Thm 1.3) & (Prop. 4.8) \\
\hline & & Matching lower bound (Thm 1.4) & Matching lower bound (Thm 1.4) \\
\hline & PSD lift & Size: $2^{n-1}$ & Size: $2 n$ \\
\hline
\end{tabular}

Table 1: Summary of results related to the convex bodies considered in the paper.

Section 3 focuses on the symmetry properties of conv $S O(n)$ and conv $O(n)$, as well as certain convex polytopes that naturally arise when studying these convex bodies. With these preliminaries established, Section 4 outlines the main arguments required to establish the correctness of the spectrahedral representations of $S O(n)^{\circ}, O(n)^{\circ}$, conv $S O(n)$ and conv $O(n)$. Details of some of the constructions required for these arguments are deferred to Appendix A. Section 5 establishes lower bounds on the size of spectrahedral representations of $S O(n)^{\circ}, O(n)^{\circ}$, conv $S O(n)$ and $\operatorname{conv} O(n)$ as well as a lower bound on the size of equivariant PSD lifts of conv $S O(n)$.

Many of the properties of the convex bodies of interest in this paper are summarized in Table 1 which may serve as a useful navigational aid when reading the paper.

\section{An illustrative application - joint satellite attitude and spin-rate estimation}

In this section we discuss a problem in satellite attitude estimation that can be reformulated as semidefinite programs using the representation of $S O(n)^{\circ}$ described in Section 1.1. Our aim, here, is to give a concrete example of situations where the semidefinite representations we describe in this paper arise naturally. The problem of interest is one of estimating the attitude (i.e. orientation) and spin-rate of a spinning satellite, and is a slight generalization of a problem posed recently by Psiaki [25]. We first focus on describing the basic attitude estimation problem in Section 2.1, before describing the joint attitude and spin-rate estimation problem in Section 2.2. 


\subsection{Attitude estimation}

The attitude of a satellite is the element of $S O(3)$ that transforms a reference coordinate system (the inertial system) in which, say, the sun is fixed, into a local coordinate system fixed with respect to the satellite's body (the body system). We are given unit vectors $x_{1}, x_{2}, \ldots, x_{T}$ (e.g., the alignment of the Earth's magnetic field, directions of landmarks such as the sun or other stars, etc.) in the inertial coordinate system, and noisy measurements $y_{1}, y_{2}, \ldots, y_{T}$ of these directions in the body coordinate system. Let $Q \in S O(3)$ denote the unknown attitude of the satellite. The aim is to estimate (in the maximum likelihood sense) $Q$ given the $y_{k}$, the $x_{k}$ and a description of the measurement noise.

The simplest noise model assumes that each $y_{k}$ is independent has a von Mises-Fisher distribution [22] (a natural family of probability distributions on the sphere) with mean $Q x_{k}$ and concentration parameter $\kappa$ i.e. its probability density function is, up to a proportionality constant that does not depend on $Q, p\left(y_{k} ; Q\right) \propto \exp \left(\kappa\left\langle y_{k}, Q x_{k}\right\rangle\right)$. Then the maximum likelihood estimate of $Q$ is found by solving

$$
\max _{Q \in S O(3)} \sum_{k=1}^{T} \kappa\left\langle y_{k}, Q x_{k}\right\rangle=\max _{Q \in S O(3)}\left\langle Q, \kappa \sum_{k=1}^{T} y_{k} x_{k}^{T}\right\rangle=\max _{Q \in \operatorname{conv} S O(3)}\left\langle Q, \kappa \sum_{k=1}^{T} y_{k} x_{k}^{T}\right\rangle .
$$

This is a probabilistic interpretation of a problem known as Wahba's problem in the astronautical literature, posed by Grace Wahba in the July 1965 SIAM Review problems and solutions section [35, Problem 65-1].

Our spectrahedral representation of conv $S O(n)$ allows us to express the optimization problem in (11) as a semidefinite program. In the astronautical literature it is common to solve this problem via the $q$-method [19] which involves parameterizing $S O(3)$ in terms of unit quaternions and solving a symmetric eigenvalue problem. Our semidefinite programming-based formulation could be thought of as a much more flexible generalization of this eigenvalue problem-based approach that works for any $n$, not just the case $n=3$.

\subsection{Joint attitude and spin-rate estimation}

A significant benefit of having a semidefinite programming-based description of a problem (such as Wahba's problem), is that it often allows us to devise semidefinite programming-based solutions to more complicated related problems by composing semidefinite representations in different ways. An example of this is given by the following generalization of Wahba's problem posed by Psiaki [25]. ${ }^{2}$

Consider a satellite rotating at a constant unknown angular velocity $\omega \mathrm{rad} / \mathrm{sample}$ around a known axis (e.g. its major axis). Assume the body coordinate system is chosen so that the rotation is around the axis defined by the first coordinate direction. Then the attitude matrix at the $k$ th sample instant is of the form

$$
Q(k)=\left[\begin{array}{ccc}
1 & 0 & 0 \\
0 & \cos (k \omega) & -\sin (k \omega) \\
0 & \sin (k \omega) & \cos (k \omega)
\end{array}\right] Q
$$

where $Q \in S O(3)$ is the initial attitude. Suppose, now, the satellite sequentially obtains measurements $y_{0}, y_{1}, \ldots, y_{T}$ in the body coordinate system of known landmarks in the directions $x_{0}, x_{1}, \ldots, x_{T}$ in the inertial coordinate system. As before assume that the $y_{k}$ are independent

\footnotetext{
${ }^{2}$ Psiaki's formulation only considers the $\kappa_{2}=0$ case, where measurements of the spin rate are not considered.
} 
and have von Mises-Fisher distribution with mean $Q(k) x_{k}$ and concentration parameter $\kappa_{1}$. Furthermore, the satellite obtains a sequence $\omega_{1}, \omega_{2}, \ldots, \omega_{T}$ of noisy measurements of the unknown constant spin rate $\omega$. Suppose the $\omega_{k}$ are independent and each $\omega_{k}$ has a von Mises distribution [22] (a natural distribution for angular-valued quantities) with mean $\omega$ and concentration parameter $\kappa_{2}$, i.e., its probability density function (up to a constant independent of $\omega$ ) is given by $p\left(\omega_{k} ; \omega\right) \propto \exp \left(\kappa_{2} \cos \left(\omega_{k}-\omega\right)\right)$. If the $\omega_{k}$ and the $y_{k}$ are independent then the maximum likelihood estimate of $Q$ and $\omega$ can be found by solving

$$
\max _{\substack{Q \in S O(3) \\
\omega \in[0,2 \pi)}} \sum_{k=0}^{T}\left\langle y_{k}, \kappa_{1}\left[\begin{array}{ccc}
1 & 0 & 0 \\
0 & \cos (k \omega) & -\sin (k \omega) \\
0 & \sin (k \omega) & \cos (k \omega)
\end{array}\right] Q x_{k}\right\rangle+\kappa_{2} \sum_{k=0}^{T} \cos \left(\omega_{k}-\omega\right) .
$$

Note that the optimization problem (12) can be rewritten as

$$
\max _{\substack{Q \in S O(3) \\ \omega \in[0,2 \pi)}} a_{1} \cos (\omega)+b_{1} \sin (\omega)+\left\langle A_{0}, Q\right\rangle+\sum_{k=1}^{T}\left\langle A_{k}, \cos (k \omega) Q\right\rangle+\left\langle B_{k}, \sin (k \omega) Q\right\rangle,
$$

i.e. the maximization of a linear functional over

$\mathcal{M}_{3, T}=\{(\cos (\omega), \sin (\omega), Q, \cos (\omega) Q, \sin (\omega) Q, \ldots, \cos (T \omega) Q, \sin (T \omega) Q): Q \in S O(3), \omega \in[0,2 \pi)\}$.

We can reformulate this as a semidefinite program if we have a PSD lift of $\operatorname{conv}\left(\mathcal{M}_{3, T}\right)$, because the optimization problem (13) is equivalent to the maximization of the same linear functional over $\operatorname{conv}\left(\mathcal{M}_{3, T}\right)$. Using the fact that $S O(n)^{\circ}$ has a spectrahedral representation of size $2^{n-1}$, it can be shown that that $\operatorname{conv}\left(\mathcal{M}_{n, T}\right)$ has a PSD lift of size $2^{n-1}(T+1)$. Describing this in detail is beyond the scope of the present paper. Instead we discuss this reformulation in further detail in a separate report [30].

\section{Basic properties of conv $S O(n)$ and conv $O(n)$}

In this section we consider the convex bodies conv $S O(n)$ and conv $O(n)$ purely from the point of view of convex geometry leaving discussion of aspects related to their semidefinite representations for Section 4. In this section we describe their symmetries, and how the full space of $\mathbb{R}^{n \times n}$ matrices decomposes with respect to these symmetries, via the (special) singular value decomposition. To a large extent one can characterize conv $S O(n)$ and conv $O(n)$ in terms of their intersections with the subspace of diagonal matrices. These diagonal sections are well known polytopes - the parity polytope and the hypercube respectively. The properties of these diagonal sections are crucial to establishing our spectrahedral representation of conv $S O(n)$ in Section 4.3 and the lower bounds on the size of spectrahedral representations given in Section 5.

All of the results in this section are (sometimes implicitly) in the literature in various forms. Here we aim for a brief yet unified presentation to make the paper as self-contained as possible.

\subsection{Symmetry and the special singular value decomposition}

In this section we describe the symmetries of $\operatorname{conv} O(n)$ and $\operatorname{conv} S O(n)$.

The group $O(n) \times O(n)$ acts on $\mathbb{R}^{n \times n}$ by $(U, V) \cdot X=U X V^{T}$. This action leaves the set $O(n)$ invariant, and hence leaves the convex bodies conv $O(n)$ and $O(n)^{\circ}$ invariant. It is also useful to understand how the ambient space of $n \times n$ matrices decomposes under this group action. Indeed 
by the well-known singular value decomposition every element $X \in \mathbb{R}^{n \times n}$ can be expressed as $X=U \Sigma V^{T}=(U, V) \cdot \Sigma$ where $(U, V) \in O(n) \times O(n)$, and $\Sigma$ is diagonal with $\Sigma_{11} \geq \cdots \geq \Sigma_{n n} \geq 0$. These diagonal elements are the singular values. We denote them by $\sigma_{i}(X)=\Sigma_{i i}$. Note that for most of what follows, we only use the fact that $\Sigma$ is diagonal, not that its elements can be taken to be non-negative and sorted.

Similarly the group

$$
S(O(n) \times O(n))=\{(U, V): U, V \in O(n), \operatorname{det}(U) \operatorname{det}(V)=1\}
$$

acts on $\mathbb{R}^{n \times n}$ by $(U, V) \cdot X=U X V^{T}$. This action leaves the sets $S O(n)$ and $S O^{-}(n)$ invariant, and hence leaves the convex bodies conv $S O(n)$, conv $S O^{-}(n), S O(n)^{\circ}, S O^{-}(n)^{\circ}, \operatorname{conv} O(n)$ and $O(n)^{\circ}$ invariant. A variant on the singular value decomposition, known as the special singular value decomposition [29] describes how the space of $n \times n$ matrices decomposes under this group action. Indeed every $X \in \mathbb{R}^{n \times n}$ can be expressed as $X=U \tilde{\Sigma} V^{T}=(U, V) \cdot \tilde{\Sigma}$ where $(U, V) \in S(O(n) \times O(n))$ and $\tilde{\Sigma}$ is diagonal with $\tilde{\Sigma}_{11} \geq \cdots \geq \tilde{\Sigma}_{n-1, n-1} \geq\left|\tilde{\Sigma}_{n n}\right|$. These diagonal elements are the special singular values. We denote them by $\tilde{\sigma}_{i}(X)=\tilde{\Sigma}_{i i}$. Again in what follows we typically only use the fact that $\tilde{\Sigma}$ is diagonal for our arguments.

The special singular value decomposition can be obtained from the singular value decomposition. Suppose $X=U \Sigma V^{T}$ is a singular value decomposition of $X$ so that $(U, V) \in O(n) \times O(n)$. If $\operatorname{det}(U) \operatorname{det}(V)=1$ this is also a valid special singular value decomposition. Otherwise, if $\operatorname{det}(U) \operatorname{det}(V)=-1$ then $X=U R(R \Sigma) V^{T}$ gives a decomposition where $(U R, V) \in S(O(n) \times O(n))$ and $R \Sigma$ is again diagonal, but with the last diagonal entry being negative. As such the singular values and special singular values of an $n \times n$ matrix are related by $\sigma_{i}(X)=\tilde{\sigma}_{i}(X)$ for $i=1,2, \ldots, n-1$ and $\tilde{\sigma}_{n}(X)=\operatorname{sign}(\operatorname{det}(X)) \sigma_{n}(X)$.

The importance of these decompositions of $\mathbb{R}^{n \times n}$ under the action of $O(n) \times O(n)$ and $S(O(n) \times$ $O(n))$ is that they allow us to reduce many arguments, by invariance properties, to arguments about diagonal matrices.

\subsection{Polytopes associated with $\operatorname{conv} O(n)$ and conv $S O(n)$}

The convex hull of $O(n)$ is closely related to the hypercube

$$
\mathrm{C}_{n}=\operatorname{conv}\left\{x \in \mathbb{R}^{n}: x_{i}^{2}=1, \text { for } i \in[n]\right\} ;
$$

the convex hull of $S O(n)$ is closely related to the parity polytope

$$
\mathrm{PP}_{n}=\operatorname{conv}\left\{x \in \mathbb{R}^{n}: \prod_{i=1}^{n} x_{i}=1, x_{i}^{2}=1, \text { for } i \in[n]\right\} ;
$$

the convex hull of $\mathrm{SO}^{-}(n)$ is closely related to the odd parity polytope

$$
\mathrm{PP}_{n}^{-}=\operatorname{conv}\left\{x \in \mathbb{R}^{n}: \prod_{i=1}^{n} x_{i}=-1 . x_{i}^{2}=1, \text { for } i \in[n]\right\} .
$$

In this section we briefly discuss these polytopes as well as showing that they are the diagonal sections of conv $O(n)$, conv $S O(n)$ and conv $S O^{-}(n)$ respectively.

Facet descriptions Irredundant descriptions of $\mathrm{C}_{n}$ and $\mathrm{PP}_{n}$ in terms of linear inequalities are well known [18]. The hypercube has $2 n$ facets corresponding to the linear inequality description

$$
\mathrm{C}_{n}=\left\{x \in \mathbb{R}^{n}:-1 \leq x_{i} \leq 1 \text { for } i \in[n]\right\}
$$


For $n \geq 4$ the parity polytope $\mathrm{PP}_{n}$ has $2 n+2^{n-1}$ facets corresponding to the linear inequality description

$$
\mathrm{PP}_{n}=\left\{x \in \mathbb{R}^{n}:-1 \leq x_{i} \leq 1 \text { for } i \in[n], \sum_{i \notin I} x_{i}-\sum_{i \in I} x_{i} \leq n-2 \text { for } I \subseteq[n],|I| \text { odd }\right\} .
$$

In the cases $n=2$ and $n=3$ this description simplifies to

$$
\begin{aligned}
& \mathrm{PP}_{2}=\left\{\left[\begin{array}{l}
x \\
x
\end{array}\right] \in \mathbb{R}^{2}:-1 \leq x \leq 1\right\} \mathrm{PP}_{3}=\left\{x \in \mathbb{R}^{3}:\right. \\
& x_{1}-x_{2}+x_{3} \leq 1,-x_{1}+x_{2}+x_{3} \leq 1, \\
&\left.x_{1}+x_{2}-x_{3} \leq 1,-x_{1}-x_{2}-x_{3} \leq 1\right\}
\end{aligned}
$$

showing that $\mathrm{PP}_{3}$ has only four facets.

The polar of the hypercube is the cross-polytope. We denote it by $\mathrm{C}_{n}^{\circ}$. It is clear from (14) that $\mathrm{C}_{n}^{\circ}$ has $2^{n}$ facets and corresponding linear inequality description

$$
\mathrm{C}_{n}^{\circ}=\left\{x \in \mathbb{R}^{n}: \sum_{i \notin I} x_{i}-\sum_{i \in I} x_{i} \leq 1 \quad \text { for } I \subset[n]\right\} .
$$

The polar of the parity polytope is denoted by $\mathrm{PP}_{n}^{\circ}$. It is clear from (15) that $\mathrm{PP}_{n}^{\circ}$ has $2^{n-1}$ facets and corresponding linear inequality description

$$
\mathrm{PP}_{n}^{\circ}=\left\{x \in \mathbb{R}^{n}: \sum_{i \notin I} x_{i}-\sum_{i \in I} x_{i} \leq 1 \quad \text { for } I \subset[n],|I| \text { even }\right\} .
$$

Similarly

$$
\mathrm{PP}_{n}^{-\circ}=\left\{x \in \mathbb{R}^{n}: \sum_{i \notin I} x_{i}-\sum_{i \in I} x_{i} \leq 1 \quad \text { for } I \subseteq[n],|I| \text { odd }\right\} .
$$

To get a sense of the importance of these polytopes for understanding conv $S O(n)$ it may be instructive to compare (19) with (9), (20) with (10), (18) with (8), and (22) with (7).

We conclude the discussion of these polytopes with a useful alternative description of $\mathrm{PP}_{n}$.

Lemma 3.1. The parity polytope can be expressed as

$$
\mathrm{PP}_{n}=\mathrm{C}_{n} \cap(n-2) \cdot \mathrm{PP}_{n}^{-\circ} \text {. }
$$

In the case $n=3$ this simplifies to $\mathrm{PP}_{3}=\mathrm{PP}_{3}^{-\circ}$.

Proof. For the general case, we need only examine the facet descriptions in (17), (18), and (23). In the case $n=3$ the result follows by comparing (20) with (23).

Diagonal projections and sections We now establish the link between the hypercube and the convex hull of $O(n)$, and the parity polytope and the convex hull of $S O(n)$. First we prove a result that says that the subspace $\mathcal{D}$ of diagonal matrices interacts particularly well with these convex bodies. The theorem applies for the convex bodies conv $O(n)$, conv $S O(n)$ and conv $S O^{-}(n)$ because whenever $g$ is a diagonal matrix with non-zero entries in $\{-1,1\}$ (a diagonal sign matrix) then each of these convex bodies is invariant under the conjugation map $X \mapsto g X g^{T}$.

Lemma 3.2. Let $C \subset \mathbb{R}^{n \times n}$ be a convex body that is invariant under conjugation by diagonal sign matrices. Then $\pi_{\mathcal{D}}(C)=\pi_{\mathcal{D}}(C \cap \mathcal{D})$ and $\left[\pi_{\mathcal{D}}(C \cap \mathcal{D})\right]^{\circ}=\pi_{\mathcal{D}}\left(C^{\circ} \cap \mathcal{D}\right)$. 
Proof. We first establish that $\pi_{\mathcal{D}}(C)=\pi_{\mathcal{D}}(C \cap \mathcal{D})$. Note that clearly $\pi_{\mathcal{D}}(C \cap \mathcal{D}) \subseteq \pi_{\mathcal{D}}(C)$. For the reverse inclusion let $G$ denote the group of diagonal sign matrices and observe that $\mathcal{D}$ is the subspace of $n \times n$ matrices fixed pointwise by the conjugation action of diagonal sign matrices. Then for any $X \in C$ the projection onto $\mathcal{D}$, the fixed point subspace, is

$$
\pi_{\mathcal{D}}^{*} \pi_{\mathcal{D}}(X)=\frac{1}{2^{n}} \sum_{g \in G} g X g^{T}
$$

which gives a description of $\pi_{\mathcal{D}}^{*} \pi_{\mathcal{D}}(X)$ as a convex combination of the $g X g^{T}$. Each $g X g^{T} \in C$ since $C$ is invariant under conjugation by diagonal sign matrices. Hence $\pi_{\mathcal{D}}^{*} \pi_{\mathcal{D}}(X) \in C \cap \mathcal{D}$ and so $\pi_{\mathcal{D}}(X) \in \pi_{\mathcal{D}}(C \cap \mathcal{D})$.

Now we establish that $\left[\pi_{\mathcal{D}}(C \cap \mathcal{D})\right]^{\circ}=\pi_{\mathcal{D}}\left(C^{\circ} \cap \mathcal{D}\right)$. For any $y \in \mathcal{D}$ we have that

$$
\max _{x \in \pi_{\mathcal{D}}(C \cap \mathcal{D})}\langle y, x\rangle=\max _{x \in \pi_{\mathcal{D}}(C)}\langle y, x\rangle=\max _{z \in C}\left\langle y, \pi_{\mathcal{D}}(z)\right\rangle=\max _{z \in C}\left\langle\pi_{\mathcal{D}}^{*}(y), z\right\rangle .
$$

Hence $y \in\left[\pi_{\mathcal{D}}(C \cap \mathcal{D})\right]^{\circ}$ if and only if $\pi_{\mathcal{D}}^{*}(y) \in C^{\circ}$, or, equivalently, $y \in \pi_{\mathcal{D}}\left(C^{\circ} \cap \mathcal{D}\right)$.

We note that this lemma generalizes to the situation where $C$ is a convex body invariant under the action of a compact group and the subspace $\mathcal{D}$ is replaced with the fixed point subspace of the group action.

The key fact that relates the parity polytope and the convex hull of $S O(n)$ is the following celebrated theorem of Horn [16].

Theorem 3.3 (Horn). The projection onto the diagonal of $S O(n)$ is the parity polytope, i.e. $\pi_{\mathcal{D}}(S O(n))=\mathrm{PP}_{n}$.

Note that we do not need the full strength of Horn's theorem. We only use the corollaries that

$$
\begin{aligned}
\pi_{\mathcal{D}}(\operatorname{conv} S O(n)) & =\operatorname{conv} \pi_{\mathcal{D}}(S O(n))=\operatorname{conv} \mathrm{PP}_{n}=\mathrm{PP}_{n} \quad \text { and } \\
\pi_{\mathcal{D}}\left(\operatorname{conv} S O^{-}(n)\right) & =\pi_{\mathcal{D}}(R \cdot \operatorname{conv} S O(n))=R \cdot \pi_{\mathcal{D}}(\operatorname{conv} S O(n))=R \cdot \mathrm{PP}_{n}=\mathrm{PP}_{n}^{-}
\end{aligned}
$$

We are now in a position to establish the main result of this section.

Proposition 3.4. Let $\mathcal{D} \subset \mathbb{R}^{n \times n}$ denote the subspace of diagonal matrices. Then

$$
\begin{array}{rlrl}
\pi_{\mathcal{D}}(\mathcal{D} \cap \operatorname{conv} O(n)) & =\mathrm{C}_{n}, & \pi_{\mathcal{D}}\left(\mathcal{D} \cap O(n)^{\circ}\right) & =\mathrm{C}_{n}^{\circ}, \\
\pi_{\mathcal{D}}(\mathcal{D} \cap \operatorname{conv} S O(n)) & =\mathrm{PP}_{n}, \quad \pi_{\mathcal{D}}\left(\mathcal{D} \cap S O(n)^{\circ}\right)=\mathrm{PP}_{n}^{\circ}, \\
\pi_{\mathcal{D}}\left(\mathcal{D} \cap \operatorname{conv} S O^{-}(n)\right) & =\mathrm{PP}_{n}^{-}, & \pi_{\mathcal{D}}\left(\mathcal{D} \cap S O^{-}(n)^{\circ}\right) & =\mathrm{PP}_{n}^{-\circ}
\end{array}
$$

Proof. First note that by (24) and (25) we know that $\pi_{\mathcal{D}}(\operatorname{conv} S O(n))=\mathrm{PP}_{n}$ and that $\pi_{\mathcal{D}}\left(\operatorname{conv} S O^{-}(n)\right)=$ $\mathrm{PP}_{n}^{-}$. Consequently

$$
\pi_{\mathcal{D}}(\operatorname{conv} O(n))=\operatorname{conv} \pi_{\mathcal{D}}\left(S O(n) \cup S O^{-}(n)\right)=\operatorname{conv}\left(\mathrm{PP}_{n} \cup \mathrm{PP}_{n}^{-}\right)=\mathrm{C}_{n} .
$$

Since each of conv $O(n)$, conv $S O(n)$, conv $S O^{-}(n)$ is invariant under conjugation by diagonal sign matrices we can apply Lemma 3.2. Doing so and using the characterization of the diagonal projections of each of these convex bodies from the previous paragraph, completes the proof. 


\section{Spectrahedral representations of $S O(n)^{\circ}$ and conv $S O(n)$}

This section is devoted to outlining the proofs of Theorems 1.1, 1.2 and 1.3, giving spectrahedral representations of $S O(n)^{\circ} O(n)^{\circ}$ and conv $S O(n)$. For the sake of exposition, we initially focus on $S O(2)^{\circ}$ as in this case all the ideas are familiar. Low dimensional coincidences do mean that some issues are simpler in the $2 \times 2$ case than in general. After discussing the $2 \times 2$ case, in Section 4.2 we generalize the argument, deferring some details to Appendix A. Finally in Section 4.3 we construct our spectrahedral representation of conv $S O(n)$.

\subsection{The $2 \times 2$ case}

We begin by giving a spectrahedral representations of $S O(2)^{\circ}$. We make crucial use of the trigonometric identities $\cos (\theta)=\cos ^{2}(\theta / 2)-\sin ^{2}(\theta / 2)$ and $\sin (\theta)=2 \cos (\theta / 2) \sin (\theta / 2)$. Recall that elements of $S O(2)$ have the form

$$
\left[\begin{array}{cc}
\cos (\theta) & -\sin (\theta) \\
\sin (\theta) & \cos (\theta)
\end{array}\right]=\left[\begin{array}{cc}
\cos ^{2}\left(\frac{\theta}{2}\right)-\sin ^{2}\left(\frac{\theta}{2}\right) & -2 \cos \left(\frac{\theta}{2}\right) \sin \left(\frac{\theta}{2}\right) \\
2 \cos \left(\frac{\theta}{2}\right) \sin \left(\frac{\theta}{2}\right) & \cos ^{2}\left(\frac{\theta}{2}\right)-\sin ^{2}\left(\frac{\theta}{2}\right)
\end{array}\right]
$$

and that $(\cos (\theta / 2), \sin (\theta / 2))$ parameterizes the unit circle in $\mathbb{R}^{2}$. Hence $S O(2)$ is the image of the unit circle $\left\{\left(x_{1}, x_{2}\right): x_{1}^{2}+x_{2}^{2}=1\right\}$ under the quadratic map

$$
Q\left(x_{1}, x_{2}\right)=\left[\begin{array}{cc}
x_{1}^{2}-x_{2}^{2} & -2 x_{1} x_{2} \\
2 x_{1} x_{2} & x_{1}^{2}-x_{2}^{2}
\end{array}\right]
$$

As such, $Y \in S O(2)^{\circ}$ if and only if, for all $\left(x_{1}, x_{2}\right)$ in the unit circle,

$$
\begin{aligned}
\left\langle Y, Q\left(x_{1}, x_{2}\right)\right\rangle & =\left\langle\left[\begin{array}{ll}
Y_{11} & Y_{12} \\
Y_{21} & Y_{22}
\end{array}\right],\left[\begin{array}{cc}
x_{1}^{2}-x_{2}^{2} & -2 x_{1} x_{2} \\
2 x_{1} x_{2} & x_{1}^{2}-x_{2}^{2}
\end{array}\right]\right\rangle \\
& =\left[\begin{array}{ll}
x_{1} & x_{2}
\end{array}\right]\left[\begin{array}{cc}
Y_{11}+Y_{22} & Y_{21}-Y_{12} \\
Y_{21}-Y_{12} & -Y_{11}-Y_{22}
\end{array}\right]\left[\begin{array}{l}
x_{1} \\
x_{2}
\end{array}\right] \leq 1 .
\end{aligned}
$$

This is equivalent to the spectrahedral representation

$$
S O(2)^{\circ}=\left\{Y:\left[\begin{array}{cc}
Y_{11}+Y_{22} & Y_{21}-Y_{12} \\
Y_{21}-Y_{12} & -Y_{11}-Y_{22}
\end{array}\right] \preceq I\right\}
$$

which coincides with the $n=2$ case of Theorem 1.1 .

To summarize, the main idea of the argument is that we use a parameterization of $S O(2)$ as the image of the unit circle under a quadratic map. This parameterization allows us to rewrite the maximum of a linear functional on $S O(2)$ as the maximum of a quadratic form on the unit circle which can be expressed as a spectrahedral condition.

We note that a very similar argument works in the case $n=3$ to directly produce the representations of $S O(3)^{\circ}$ and conv $S O(3)$ in Theorem 1.1 and Corollary 1.6 respectively. Indeed the unit quaternion parameterization of rotations gives a parameterization of $S O(3)$ as the image of the unit sphere in $\mathbb{R}^{4}$ under a quadratic mapping again allowing us to rewrite the maximum of a linear functional on $S O(3)$ as the maximum of a quadratic form on the unit sphere which is equivalent to a spectrahedral condition. 


\subsection{Outline of the general argument}

For the general case, we first need a quadratic parameterization of $S O(n)$. There is a classical construction of a quadratic map $Q: \mathbb{R}^{2^{n-1}} \rightarrow \mathbb{R}^{n \times n}$ and a subset $\operatorname{Spin}(n)$ of the unit sphere in $\mathbb{R}^{2^{n-1}}$ such that $S O(n)=Q(\operatorname{Spin}(n))$. (We recall this construction in Appendix A, only discussing those aspects relevant for our argument here.)

Unfortunately, for $n \geq 4, \operatorname{Spin}(n)$ is a strict subset of the unit sphere in $\mathbb{R}^{2^{n-1}}$, so we cannot simply follow the argument for the $n=2$ case verbatim. The key difficulty is that we need a spectrahedral characterization of the maximum over $\operatorname{Spin}(n)$ of the quadratic form $x \mapsto\langle Y, Q(x)\rangle$ (for arbitrary $Y$ ). It is not obvious how to do this when $\operatorname{Spin}(n)$ is a strict subset of the sphere.

We achieve this by showing that for any $Y$, the maximum of the quadratic form $x \mapsto\langle Y, Q(x)\rangle$ over the entire sphere coincides with its maximum over the strict subset $\operatorname{Spin}(n)$ of the sphere (see Proposition 4.5, to follow). To establish this we exploit additional structure in $\operatorname{Spin}(n)$ and certain equivariance properties of $Q$. The specific properties we use are stated in Propositions 4.1, 4.2, 4.3, and 4.4. We prove these in Appendix A.

Proposition 4.1. There is a $2^{n-1}$-dimensional inner product space, $\mathrm{Cl}^{0}(n)$, a subset $\operatorname{Spin}(n)$ of the unit sphere in $\mathrm{Cl}^{0}(n)$ and a quadratic map $Q: \mathrm{Cl}^{0}(n) \rightarrow \mathbb{R}^{n \times n}$ such that $Q(\operatorname{Spin}(n))=S O(n)$.

Given an orthonormal basis $e_{1}, \ldots, e_{n}$ for $\mathbb{R}^{n}$ there is a corresponding orthonormal basis $\left(e_{I}\right)_{I \in \mathcal{I}_{\text {even }}}$ for $\mathrm{Cl}^{0}(n)$ indexed by $\mathcal{I}_{\text {even }}$, the subsets of $\{1,2, \ldots, n\}$ of even cardinality. This basis has the following important property.

Proposition 4.2. Each of the $2^{n-1}$ elements of the basis $\left(e_{I}\right)_{I \in \mathcal{I}_{\text {even }}}$ is in $\operatorname{Spin}(n)$.

For the rest of this section we fix these two choices of basis for $\mathbb{R}^{n}$ and $\mathrm{Cl}^{0}(n)$ respectively. With respect to the basis $\left(e_{I}\right)_{I \in \mathcal{I}_{\text {even }}}$ we write $x \in \mathrm{Cl}^{0}(n)$ in coordinates as $x=\sum_{I \in \mathcal{I}_{\text {even }}} x_{I} e_{I}$. The following result summarizes the properties of $Q(x)$, with respect to these choices of basis, that we use.

Proposition 4.3. The matrix entry functions $[Q(\cdot)]_{i j}: \mathrm{Cl}^{0}(n) \rightarrow \mathbb{R}$ are explicitly given by

$$
[Q(x)]_{i j}=\left\langle x, A_{i j} x\right\rangle=\sum_{I, J \in \mathcal{I}_{\text {even }}}\left[A_{i j}\right]_{I, J} x_{I} x_{J}
$$

where the $A_{i j}$ are the signed permutation matrices defined in (6). In particular, the quadratic forms $[Q(x)]_{i i}$ are diagonal with respect to the basis $\left(e_{I}\right)_{I \in \mathcal{I}_{\text {even }}}$.

Finally, $Q$ interacts well with left and right multiplication by elements of $S O(n)$.

Proposition 4.4. For any $U, V \in S O(n)$ there is a linear map $\Phi_{(U, V)}: \mathrm{Cl}^{0}(n) \rightarrow \mathrm{Cl}^{0}(n)$ such that

- $U Q(x) V^{T}=Q\left(\Phi_{(U, V)} x\right)$ for all $x \in \mathrm{Cl}^{0}(n)$

- $\Phi_{(U, V)}$ is invertible

- $\Phi_{(U, V)}$ and $\Phi_{(U, V)}^{-1}$ preserve $\operatorname{Spin}(n)$, i.e., $\Phi_{(U, V)}(\operatorname{Spin}(n))=\Phi_{(U, V)}^{-1}(\operatorname{Spin}(n))=\operatorname{Spin}(n)$.

The following proposition, the crux of our argument, implies that for any $n \times n$ matrix $Y$, the maximum of the quadratic form $x \mapsto\langle Y, Q(x)\rangle$ quadratic form over the whole sphere and over the (strict) subset $\operatorname{Spin}(n)$, coincide.

Proposition 4.5. Given any $Y \in \mathbb{R}^{n \times n}$ the quadratic form $x \mapsto\langle Y, Q(x)\rangle$ has a basis of eigenvectors that are elements of $\operatorname{Spin}(n)$. 
Proof. Suppose $Y \in \mathbb{R}^{n \times n}$ is arbitrary. Then by the special singular value decomposition $Y$ can be expressed as $Y=U^{T} D V$ where $U$ and $V$ are in $S O(n)$ and $D$ is diagonal. Then by Proposition 4.4

$$
\langle Y, Q(x)\rangle=\left\langle U^{T} D V, Q(x)\right\rangle=\left\langle D, U Q(x) V^{T}\right\rangle=\left\langle D, Q\left(\Phi_{(U, V)} x\right)\right\rangle .
$$

Consider the quadratic form $z \mapsto\langle D, Q(z)\rangle$. Observe that

$$
\langle D, Q(z)\rangle=\sum_{i=1}^{n} D_{i i}[Q(z)]_{i i}=\left\langle z,\left[\sum_{i=1}^{n} D_{i i} A_{i i}\right] z\right\rangle
$$

and (by Proposition 4.3) each of the $A_{i i}$ is diagonal. Hence $\sum_{i=1}^{n} D_{i i} A_{i i}$ is diagonal and so $z \mapsto$ $\langle D, Q(z)\rangle$ has $\left(e_{I}\right)_{I \in \mathcal{I}_{\text {even }}}$ as a basis of eigenvectors. Hence the quadratic form $x \mapsto\langle Y, Q(x)\rangle$ has

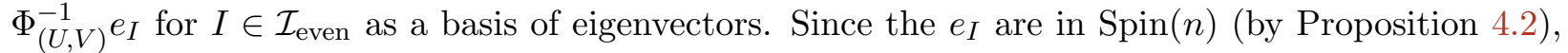
$\Phi_{(U, V)}$ is invertible, and $\Phi_{(U, V)}^{-1}$ preserves $\operatorname{Spin}(n)$ (by Proposition 4.4) we can conclude that the quadratic form $x \mapsto\langle Y, Q(x)\rangle$ has a basis of eigenvectors all of which are elements of $\operatorname{Spin}(n)$.

Assuming Propositions 4.1 and 4.5 we can prove Theorem 1.1 using an embellishment of the same argument we used in the $2 \times 2$ case.

Proof of Theorem 1.1. Since the image of $\operatorname{Spin}(n)$ under $Q$ is $S O(n)$, an $n \times n$ matrix $Y$ is in $S O(n)^{\circ}$ if and only if

$$
\max _{X \in S O(n)}\langle Y, X\rangle=\max _{x \in \operatorname{Spin}(n)}\langle Y, Q(x)\rangle \leq 1 .
$$

Since $\operatorname{Spin}(n)$ is a subset of the unit sphere in $\mathrm{Cl}^{0}(n)$, we have that

$$
\max _{x \in \operatorname{Spin}(n)}\langle Y, Q(x)\rangle \leq \max _{\substack{x \in \mathrm{Cl}^{0}(n) \\\langle x, x\rangle=1}}\langle Y, Q(x)\rangle
$$

The maximum of the quadratic form $x \mapsto\langle Y, Q(x)\rangle$ over the unit sphere in $\mathrm{Cl}^{0}(n)$ occurs at any eigenvector corresponding to the largest eigenvalue of the quadratic form. By Proposition 4.5 we can always find such an eigenvector in $\operatorname{Spin}(n)$, establishing that

$$
\max _{x \in \operatorname{Spin}(n)}\langle Y, Q(x)\rangle=\max _{\substack{x \in \mathrm{Cl}^{0}(n) \\\langle x, x\rangle=1}}\langle Y, Q(x)\rangle
$$

Hence $Y \in S O(n)^{\circ}$ if and only if for all $x \in \mathrm{Cl}^{0}(n)$ such that $\langle x, x\rangle=1$,

$$
\langle Y, Q(x)\rangle=\left\langle x, \sum_{i, j=1}^{n} Y_{i j} A_{i j} x\right\rangle \leq 1 .
$$

This is equivalent to the spectrahedral representation given in Theorem 1.1.

Remark 4.6. We briefly describe a more geometric dual interpretation of the arguments that establish Theorem 1.1. Throughout this remark let $S=\left\{x \in \mathrm{Cl}^{0}(n):\langle x, x\rangle=1\right\}$ be the unit sphere in $\mathrm{Cl}^{0}(n)$. We have seen that there is a quadratic map $Q$ such that $S O(n)=Q(\operatorname{Spin}(n)) \subset Q(S)$ with the inclusion being strict for $n \geq 4$. The remainder of the proof of Theorem 1.1 shows, from this viewpoint, that $\operatorname{conv} S O(n)=\operatorname{conv} Q(\operatorname{Spin}(n))=\operatorname{conv} Q(S)$, i.e. all the points in $S$ that are not in $\operatorname{Spin}(n)$ are mapped by $Q$ inside the convex hull of $Q(\operatorname{Spin}(n))$. One may wonder whether $Q(S)=$ conv $S O(n)$, i.e. whether the image of the sphere under $Q$ is actually convex. This is not the case - already for $n=2$ we can see that $Q(S)=S O(2) \neq \operatorname{conv} S O(2)$. 
It is now straightforward to prove Theorem 1.2, giving a spectrahedral representation of $O(n)^{\circ}$ of size $2^{n}$.

Proof of Theorem 1.2. Since $O(n)^{\circ}=S O(n)^{\circ} \cap S O^{-}(n)^{\circ}$ (see (3)) and we have already constructed a spectrahedral representation of $S O(n)^{\circ}$, it remains to give a spectrahedral representation of $S O^{-}(n)^{\circ}$. Since $S O^{-}(n)=R \cdot S O(n)$ where $R=\operatorname{diag}(1,1, \ldots, 1,-1)$, it follows that $Y \in S O^{-}(n)^{\circ}$ if and only if $\langle Y, R X\rangle=\langle R Y, X\rangle \leq 1$ for all $X \in S O(n)$. Hence $Y \in S O^{-}(n)^{\circ}$ if and only if $R Y \in S O(n)^{\circ}$.

From these observations and Theorem 1.1 we have that

$$
O(n)^{\circ}=S O(n)^{\circ} \cap S O^{-}(n)^{\circ}=\left\{Y \in \mathbb{R}^{n \times n}: \sum_{i, j=1}^{n} Y_{i j} A_{i j} \preceq I, \sum_{i, j=1}^{n}[R Y]_{i j} A_{i j} \preceq I\right\}
$$

which is a spectrahedral representation of size $2^{n}$.

\subsection{A spectrahedral representation of conv $S O(n)$}

In this section we give a spectrahedral representation of conv $S O(n)$ using a description of conv $S O(n)$ which is inherited from the corresponding description of the parity polytope.

Proposition 4.7. The convex hull of $S O(n)$ can be expressed in terms of $\operatorname{conv} O(n)$ and $S O(n)^{\circ}$ as

$$
\operatorname{conv} S O(n)=\operatorname{conv} O(n) \cap(n-2) S O^{-}(n)^{\circ} .
$$

If $n=3$ this simplifies to $\operatorname{conv} S O(3)=S O^{-}(3)^{\circ}$.

Proof. Suppose $X \in \mathbb{R}^{n \times n}$ is arbitrary. By the special singular value decomposition $X=U \tilde{\Sigma} V^{T}$ where $(U, V) \in S(O(n) \times O(n))$ and $\tilde{\Sigma}=\operatorname{diag}^{*}(\tilde{\sigma})$ is diagonal. Then since $S O(n)$ is invariant under the action of $S(O(n) \times O(n))$, it follows that $X \in \operatorname{conv} S O(n)$ if and only if $\tilde{\Sigma} \in$ conv $S O(n) \cap \mathcal{D}$. Similarly since conv $O(n)$ and $S O^{-}(n)^{\circ}$ are invariant under the action of $S(O(n) \times O(n))$, it follows that $X \in \operatorname{conv} O(n) \cap(n-2) S O^{-}(n)^{\circ}$ if and only if $\tilde{\Sigma} \in \operatorname{conv} O(n) \cap \mathcal{D}$ and $\tilde{\Sigma} \in(n-2) S O^{-}(n)^{\circ} \cap \mathcal{D}$.

Since the diagonal section of conv $S O(n)$ is the parity polytope, $X \in \operatorname{conv} S O(n)$ if and only if $\tilde{\sigma} \in \mathrm{PP}_{n}$. Since the diagonal section of conv $O(n)$ is the hypercube, $\tilde{\sigma} \in \mathrm{C}_{n}$ if and only if $\tilde{\Sigma} \in \operatorname{conv} O(n) \cap \mathcal{D}$. Since the diagonal section of $S O^{-}(n)^{\circ}$ is $\mathrm{PP}_{n}^{-\circ}, \tilde{\sigma} \in(n-2) \mathrm{PP}_{n}^{-\circ}$ if and only if $\tilde{\Sigma} \in(n-2) S O^{-}(n)^{\circ} \cap \mathcal{D}$.

Finally we use the fact that $\mathrm{PP}_{n}=\mathrm{C}_{n} \cap(n-2) \mathrm{PP}_{n}^{-\circ}$ (see Lemma 3.1). Then $X \in \operatorname{conv} S O(n)$ if and only if $\tilde{\sigma} \in \mathrm{PP}_{n}$ which occurs if and only if $\tilde{\sigma} \in \mathrm{C}_{n}$ and $\tilde{\sigma} \in(n-2) \mathrm{PP}_{n}^{-\circ}$ which occurs if and only if $X \in \operatorname{conv} O(n) \cap(n-2) S O^{-}(n)^{\circ}$.

In the case $n=3$ the description $\mathrm{PP}_{n}=\mathrm{C}_{n} \cap(n-2) \mathrm{PP}_{n}^{-\circ}$ simplifies to $\mathrm{PP}_{3}=\mathrm{PP}_{3}^{-\circ}$. The corresponding simplification propagates through the above argument to give conv $S O(3)=S^{-}(3)^{\circ}$.

Since the description of conv $S O(n)$ in Proposition 4.7 involves conv $O(n)$, we first give the well-known spectrahedral representation of conv $O(n)$.

Proposition 4.8. The convex hull of $O(n)$ is a spectrahedron. An explicit spectrahedral representation of size $2 n$ is given by

$$
\operatorname{conv} O(n)=\left\{X \in \mathbb{R}^{n \times n}:\left[\begin{array}{cc}
0 & X \\
X^{T} & 0
\end{array}\right] \preceq I_{2 n}\right\}
$$


Proof. Let $Q \in O(n)$ be arbitrary. Then since $Q^{T} Q=I_{n}$ it follows that

$$
\left[\begin{array}{cc}
I_{n} & -Q \\
-Q^{T} & I_{n}
\end{array}\right]=\left[\begin{array}{c}
I_{n} \\
-Q^{T}
\end{array}\right]\left[\begin{array}{ll}
I_{n} & -Q
\end{array}\right] \succeq 0
$$

and so $Q$ is an element of the right hand side of (27). Since the right hand side of (27) is convex, it follows that conv $O(n) \subseteq\left\{X \in \mathbb{R}^{n \times n}:\left[\begin{array}{cc}0 & X \\ X^{T} & 0\end{array}\right] \preceq I_{2 n}\right\}$.

For the reverse inclusion, suppose $X$ is an element of the right hand side of (27). By the singular value decomposition there is a diagonal matrix $\Sigma$ such that $X=U \Sigma V^{T}$ where $U, V \in O(n)$. Conjugating by the orthogonal matrix $\left[\begin{array}{cc}U^{T} & 0 \\ 0 & V^{T}\end{array}\right]$ we see that

$$
\left[\begin{array}{cc}
0 & X \\
X^{T} & 0
\end{array}\right] \preceq I_{2 n} \Longleftrightarrow\left[\begin{array}{cc}
0 & \Sigma \\
\Sigma & 0
\end{array}\right] \preceq I_{2 n}
$$

which is equivalent to $-1 \leq \Sigma_{i i} \leq 1$ for $i \in[n]$. Since $\mathcal{D} \cap \operatorname{conv} O(n)$ is the hypercube it follows that $\Sigma \in \mathcal{D} \cap \operatorname{conv} O(n)$ and so that $U \Sigma V^{T} \in \operatorname{conv} O(n)$.

Proof of Theorem 1.3. Since we now have a spectrahedral representation of $\operatorname{conv} O(n)$ (from (27)) and of $S O^{-}(n)^{\circ}$ (from (26)), we can use Proposition 4.7 to combine them to give the spectrahedral representation

$$
\operatorname{conv} S O(n)=\left\{X \in \mathbb{R}^{n \times n}:\left[\begin{array}{cc}
0 & X \\
X^{T} & 0
\end{array}\right] \preceq I_{2 n}, \quad \sum_{i, j=1}^{n} A_{i j}[R X]_{i j} \preceq(n-2) I_{2^{n-1}}\right\} .
$$

In the case $n=3$ Proposition 4.7 tells us that conv $S O(3)=S O^{-}(3)^{\circ}$ and so

$$
\operatorname{conv} S O(3)=\left\{X \in \mathbb{R}^{3 \times 3}: \sum_{i, j=1}^{3} A_{i j}[R X]_{i j} \preceq I_{4}\right\}
$$

which can be expressed explicitly as in (10) by using the definition of the $A_{i j}$ in (6).

To conclude the proof we explicitly simplify the spectrahedral representation (8) for the case $n=2$. Indeed

$$
\operatorname{conv} S O(2)=\left\{X \in \mathbb{R}^{2 \times 2}:\left[\begin{array}{cc}
I & -X \\
-X^{T} & I
\end{array}\right] \succeq 0,\left[\begin{array}{cc}
-X_{11}+X_{22} & -X_{21}-X_{12} \\
-X_{21}-X_{12} & X_{11}-X_{22}
\end{array}\right] \preceq 0\right\} .
$$

Since $\left[\begin{array}{cc}-X_{11}+X_{22} & -X_{21}-X_{12} \\ -X_{21}-X_{12} & X_{11}-X_{22}\end{array}\right]$ has trace zero, if it is also negative semidefinite then it must actually be zero. Consequently if $X \in \operatorname{conv} S O(2)$ then it must satisfy $X_{11}=X_{22}$ and $X_{12}=-X_{21}$ and so be of the form $X=\left[\begin{array}{cc}c & -s \\ s & c\end{array}\right]$ for some $c, s \in \mathbb{R}$. Hence

$$
\operatorname{conv} S O(2)=\left\{\left[\begin{array}{cc}
c & -s \\
s & c
\end{array}\right] \in \mathbb{R}^{2 \times 2}:\left[\begin{array}{cccc}
1 & 0 & -c & s \\
0 & 1 & -s & -c \\
-c & -s & 1 & 0 \\
s & -c & 0 & 1
\end{array}\right] \succeq 0\right\} .
$$

This is still a spectrahedral representation of size 4, but the constraint has symmetry - it is invariant under simultaneously reversing the order of the rows and columns - suggesting that it can be block diagonalized [11]. Under the change of coordinates

$$
\frac{1}{2}\left[\begin{array}{cccc}
1 & 0 & -1 & 0 \\
0 & 1 & 0 & 1 \\
0 & 1 & 0 & -1 \\
-1 & 0 & -1 & 0
\end{array}\right]\left[\begin{array}{cccc}
1 & 0 & -c & s \\
0 & 1 & -s & -c \\
-c & -s & 1 & 0 \\
s & -c & 0 & 1
\end{array}\right]\left[\begin{array}{cccc}
1 & 0 & -1 & 0 \\
0 & 1 & 0 & 1 \\
0 & 1 & 0 & -1 \\
-1 & 0 & -1 & 0
\end{array}\right]^{T}=\left[\begin{array}{cccc}
1+c & s & 0 & 0 \\
s & 1-c & 0 & 0 \\
0 & 0 & 1+c & s \\
0 & 0 & s & 1-c
\end{array}\right]
$$


we see that the size 4 spectrahedral representation in (28) is actually two copies of the same size 2 representation, allowing us to conclude that

$$
\operatorname{conv} S O(2)=\left\{\left[\begin{array}{cc}
c & -s \\
s & c
\end{array}\right] \in \mathbb{R}^{2 \times 2}:\left[\begin{array}{cc}
1+c & s \\
s & 1-c
\end{array}\right] \succeq 0\right\}
$$

as stated in Theorem 1.3.

\section{Lower bounds on the size of representations}

\subsection{Spectrahedral representations}

Whenever a convex set has a polyhedral section, we can immediately obtain a simple lower bound on the possible size of a spectrahedral representation of that convex set in terms of the number of facets of that polyhedron.

Lemma 5.1. Suppose $C \subset \mathbb{R}^{n}$ has a spectrahedral representation of size $m$ and $V$ is a subspace of $\mathbb{R}^{n}$ such that $C \cap V$ is a polytope with $f$ (irredundant) facets. Then $m \geq f$.

Proof. Suppose $C$ has a spectrahedral representation $C=\left\{x: \sum_{i} A_{i} x_{i}+A_{0} \succeq 0\right\}$ of size $m$, so the matrices $A_{i}$ are $m \times m$. Then $p(x)=\operatorname{det}\left(\sum_{i} A_{i} x_{i}+A_{0}\right)$ is a polynomial of degree at most $m$ that vanishes on the boundary of $C$. If $V$ is any subspace of $\mathbb{R}^{n}$ then $\left.p\right|_{V}$ is a polynomial of degree at most the degree of $p$ that vanishes on the boundary of $C \cap V$. Finally, any polynomial that vanishes on the boundary of a polyhedron with $f$ (irredundant) facets has degree at least $f$ (since it must have a linear factor for each facet-defining hyperplane). Consequently we have the chain of inequalities

$$
f \leq \operatorname{deg}\left(\left.p\right|_{V}\right) \leq \operatorname{deg}(p) \leq m
$$

establishing the result.

Remarkably this simple technique allows us to establish that our spectrahedral representations are of minimum size.

Proof of Theorem 1.4. The diagonal slice of $O(n)^{\circ}$ is the cross-polytope, which (for $n \geq 1$ ) has $2^{n}$ facets. Hence, for $n \geq 1$, any spectrahedral representation of $O(n)^{\circ}$ has size at least $2^{n}$. The diagonal slice of $S O(n)^{\circ}$ is the polar of the parity polytope, which (for $n \geq 2$ ) has $2^{n-1}$ facets. Hence, for $n \geq 2$, any spectrahedral representation of $S O(n)^{\circ}$ has size at least $2^{n-1}$. The diagonal slice of conv $S O(n)$ is the parity polytope, which for $n \geq 4$ has $2^{n-1}+2 n$ facets, and for $n=3$ has 4 facets. It follows that any spectrahedral representation of conv $S O(n)$ has size at least $2^{n-1}+2 n$ for $n \geq 4$ and size at least 4 for $n=3$.

The spectrahedral representations we construct in Section 4 achieve these lower bounds and so are of minimum size.

\subsection{Equivariant PSD lifts}

As is established in Theorem 1.4, our spectrahedral representations are necessarily of exponential size. While they are useful in practice for very small $n$ (such as the physically relevant $n=3$ case), this is not the case for larger $n$. 
PSD lifts In general if $C$ is a spectrahedron, it may be possible to give a much smaller projected spectrahedral representation of $C$. In other words, it may be the case that $C=\pi(D)$ where $\pi$ is a linear map $^{3}$ and $D$ has a spectrahedral representation that has much smaller size then any spectrahedral representation of $C$. Note that throughout this section if $D$ has a spectrahedral representation of size $m$ we express it as $D=\pi\left(L \cap \mathcal{S}_{+}^{m}\right)$ where $L$ is an affine subspace of $\mathcal{S}^{m}$, the space of $m \times m$ symmetric matrices, and $\mathcal{S}_{+}^{m} \subset \mathcal{S}^{m}$ is the cone of positive semidefinite $m \times m$ symmetric matrices. The following definition is a specialization of [13, Definition 2.1].

Definition 5.2. Suppose $C \subset \mathbb{R}^{n}$ is a convex body. If $C=\pi\left(L \cap \mathcal{S}_{+}^{m}\right)$ where $L$ is an affine subspace of $m \times m$ symmetric matrices and $\pi: \mathcal{S}^{m} \rightarrow \mathbb{R}^{n}$ is a linear map, we say that $C$ has a PSD lift of size $m$.

It is straightforward to show that if $C$ has a PSD lift of size $m$, then $C^{\circ}$ also has a PSD lift of size $m$ [13]. This simple observation already yields examples of convex bodies for which there is an exponential gap between the size of the smallest spectrahedral representation and the size of the smallest PSD lift. For instance, as demonstrated in Example 1.5, the smallest possible spectrahedral representation of $O(n)^{\circ}$ has size $2^{n}$ and yet it has a PSD lift of size $2 n$.

Equivariant PSD lifts While there has been some recent progress in obtaining lower bounds on the size of PSD lifts of some polytopes $[14,5]$, little is understood about lower bounds on the size of PSD lifts of convex bodies in general. Recently, new techniques have been developed for obtaining lower bounds on the size of equivariant PSD lifts of orbitopes. These are PSD lifts that 'respect' (in a precise sense to be defined below) the symmetries of that orbitope.

In the remainder of this section we show that any projected spectrahedral representation of conv $S O(n)$ that is equivariant with respect to the action of $S(O(n) \times O(n))$, must have size exponential in $n$. The argument works by showing that from any PSD lift of conv $S O(n)$ that is equivariant with respect to the action of $S(O(n) \times O(n))$ we can construct a PSD lift of the parity polytope that is equivariant with respect to a certain group action on $\mathbb{R}^{n}$. We then apply a recent result that gives an exponential lower bound on the size of appropriately equivariant PSD lifts of the parity polytope.

The following definition [8, Definition 2] makes the notion of equivariant PSD lift precise.

Definition 5.3. Let $C \subset \mathbb{R}^{n}$ be a convex body invariant under the action of a group $G$ by linear transformations. Assume $C=\pi\left(L \cap \mathcal{S}_{+}^{m}\right)$ is a PSD lift of $C$ of size $m$. The lift is called $G$-equivariant if there is a group homomorphism $\rho: G \rightarrow G L(m)$ such that

$$
\rho(g) X \rho(g)^{T} \in L \quad \forall g \in G, \text { for all } X \in L
$$

and

$$
\pi\left(\rho(g) X \rho(g)^{T}\right)=g \cdot \pi(X) \text { for all } g \in G \text { and all } X \in L \cap \mathcal{S}_{+}^{d} .
$$

In the present setting we are interested in two particular cases of equivariant PSD lifts: $S(O(n) \times$

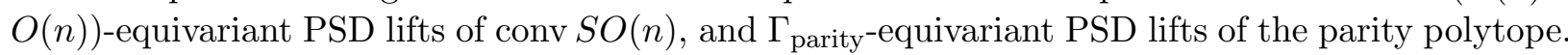
Here $\Gamma_{\text {parity }}$ can be thought of concretely as the group of evenly signed permutation matricessigned permutation matrices where there are an even number of entries that take the value -1 . These act on $\mathbb{R}^{n}$ by matrix multiplication.

We are now in a position to relate $S(O(n) \times O(n))$-equivariant PSD lifts of conv $S O(n)$ with

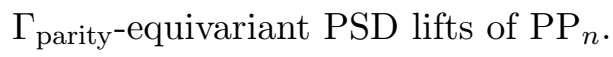

\footnotetext{
${ }^{3}$ In this section only, to conform with standard notation for PSD lifts, we use $\pi$ to mean an arbitrary linear map
} 
Proposition 5.4. If conv $S O(n)$ has an equivariant $P S D$ lift of size $m$ then $\mathrm{PP}_{n}$ has an equivariant PSD lift of size $m$.

Proof. Suppose conv $S O(n)=\pi\left(L \cap \mathcal{S}_{+}^{m}\right)$ is a $S(O(n) \times O(n))$-equivariant PSD lift of conv $S O(n)$ of size $m$ and let $\rho: S(O(n) \times O(n)) \rightarrow G L(m)$ be the associated homomorphism. Since the projection of conv $S O(n)$ onto the subspace of diagonal matrices is $\mathrm{PP}_{n}$ (Theorem 3.3) it follows that

$$
\mathrm{PP}_{n}=\left(\pi_{\mathcal{D}} \circ \pi\right)\left(L \cap \mathcal{S}_{+}^{m}\right)
$$

is a PSD lift of $\mathrm{PP}_{n}$ of size $m$. It remains to show that this lift of $\mathrm{PP}_{n}$ is $\Gamma_{\text {parity }}$-equivariant. In other words we need to construct a homomorphism $\tilde{\rho}: \Gamma_{\text {parity }} \rightarrow G L(m)$ satisfying the requirements of Definition 5.3.

First observe that any element of $\Gamma_{\text {parity }}$ can be uniquely expressed as as $D P$ where $D$ is a diagonal sign matrix with determinant one, and $P$ is a permutation matrix. Furthermore, note that if $D_{1} P_{1}$ and $D_{2} P_{2}$ are elements of $\Gamma_{\text {parity }}$, then

$$
\left(D_{1} P_{1}\right)\left(D_{2} P_{2}\right)=\left(D_{1} P_{1} D_{2} P_{1}^{T}\right)\left(P_{1} P_{2}\right)
$$

gives the associated factorization of the product. Hence define $\phi: \Gamma_{\text {parity }} \rightarrow S(O(n) \times O(n))$ by $\phi(D P)=(D P, P)$. Observe that this is a homomorphism because

$$
\phi\left(\left(D_{1} P_{1}\right)\left(D_{2} P_{2}\right)\right)=\phi\left(\left(D_{1} P_{1} D_{2} P_{1}^{T}\right)\left(P_{1} P_{2}\right)\right)=\left(\left(D_{1} P_{1}\right)\left(D_{2} P_{2}\right), P_{1} P_{2}\right)=\phi\left(D_{1} P_{1}\right) \cdot \phi\left(D_{2} P_{2}\right) .
$$

Define a homomorphism $\tilde{\rho}: \Gamma_{\text {parity }} \rightarrow G L(m)$ by $\tilde{\rho}=\rho \circ \phi$. For any symmetric matrix $X$ it is the case that $D P \cdot \pi_{\mathcal{D}}(X)=\pi_{\mathcal{D}}\left(D P X P^{T}\right)$. Hence

$$
\begin{aligned}
D P \cdot \pi_{\mathcal{D}}(\pi(X)) & =\pi_{\mathcal{D}}\left(D P \pi(X) P^{T}\right) \\
& =\pi_{\mathcal{D}}(\phi(D P, P) \cdot \pi(X)) \\
& =\pi_{\mathcal{D}}\left(\pi\left(\rho(D P, P) X \rho(D P, P)^{T}\right)\right) \quad \text { since the lift of conv } S O(n) \text { is equivariant } \\
& =\pi_{\mathcal{D}}\left(\pi\left(\tilde{\rho}(D P) X \tilde{\rho}(D P)^{T}\right)\right) \quad \text { by the definition of } \tilde{\rho}
\end{aligned}
$$

establishing that the lift is $\Gamma_{\text {parity-equivariant. }}$

The following lower bound on the size of $\Gamma_{\text {parity-equivariant PSD lifts of the parity polytope is }}$ one of the main results of [8].

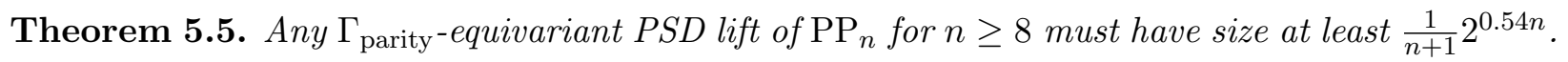

Combining Proposition 5.4 with Proposition 5.5 we obtain the following exponential lower bound on the size of any equivariant PSD lift of conv $S O(n)$.

Corollary 5.6. Any $S(O(n) \times O(n))$-equivariant $P S D$ lift of conv $S O(n)$ for $n \geq 8$ must have size at least $\frac{1}{n+1} 2^{0.54 n}$.

\section{$6 \quad$ Summary and open questions}

In this work we have constructed minimum-size spectrahedral representations for the convex hull of $S O(n)$ and its polar. We have also constructed a minimum-size spectrahedral representation of $O(n)^{\circ}$ (the nuclear norm ball). We conclude the paper by discussing some natural questions raised by our results. 


\subsection{Doubly spectrahedral convex sets}

We have seen that both the convex hull of $S O(n)$ and its polar are spectrahedra. The same is true of the convex hull of $O(n)$ (the operator norm ball) and its polar (the nuclear norm ball), as established by Sanyal et al. [29, Corollary 4.9]. This is a very special phenomenon - the polar of a spectrahedron is not, in general, a spectrahedron. For example, the intersection of the second-order cone $\left\{(x, y, z): z \geq \sqrt{x^{2}+y^{2}}\right\}$ and the non-negative orthant is a spectrahedron, but its polar has non-exposed faces and so is not a spectrahedron [26].

If a convex set $C$ and its polar are both spectrahedra, we say that $C$ is a doubly spectrahedral convex set. Apart from conv $O(n)$ and conv $S O(n)$, two distinct families of doubly spectrahedral convex sets are the following:

Polyhedra Every polyhedron is a spectrahedron, and the polar of a polyhedron is again a polyhedron. Hence polyhedra are doubly spectrahedral.

Homogeneous cones A convex cone $K$ is homogeneous if the automorphism group of $K$ acts transitively on the interior of $K$. Using Vinberg's classification of homogeneous cones in terms of $T$-algebras [34], Chua gave spectrahedral representations for all homogeneous cones [7]. Furthermore, $K$ is homogeneous if and only its dual cone $K^{*}=-K^{\circ}$ is homogeneous [34, Proposition 9]. From these two observations it follows that any homogeneous cone is doubly spectrahedral.

We have seen that the doubly spectrahedral convex sets are a strict subset of all spectrahedra that includes all polyhedra, all homogeneous convex cones, and conv $O(n)$ and conv $S O(n)$.

Problem Characterize doubly spectrahedral convex sets.

\subsection{Non-equivariant PSD lifts}

In Section 5 we showed that our spectrahedral representations of conv $S O(n)$ and $S O(n)^{\circ}$ are necessarily of exponential size and that any $S(O(n) \times O(n))$-equivariant PSD lift of conv $S O(n)$ must also have exponential size. Our lower bound on the size of $S(O(n) \times O(n))$-equivariant PSD

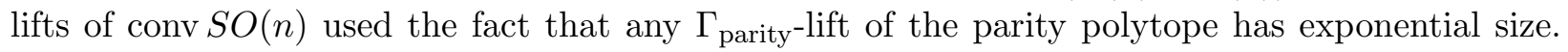
Nevertheless, the parity polytope is known to have a PSD lift (in fact it is an LP lift) of size $4(n-1)$

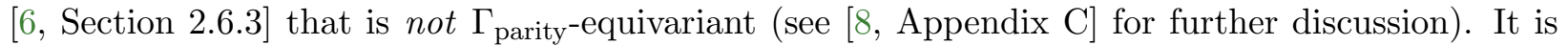
quite possible that by appropriately breaking symmetry we can find a small PSD lift of conv $S O(n)$.

Question Does conv $S O(n)$ have a PSD lift with size polynomial in $n$ ?

\section{A Clifford algebras and $\operatorname{Spin}(n)$}

In this section we describe and establish the key properties of the quadratic mapping $Q$ from Proposition 4.1 that underlies our spectrahedral representation of $S O(n)^{\circ}$ given in Theorem 1.1. The mapping $Q$ is most naturally described in terms of an algebraic structure known as a Clifford algebra, which generalizes some properties of complex numbers and quaternions. The first part of this section is devoted to describing the basic properties of Clifford algebras we require. In Section A.2 we describe the mapping $Q$ and some of its properties. In Section A.3 we define the set $\operatorname{Spin}(n)$ and establish enough of its properties to prove Propositions 4.1, 4.2, and 4.4. We prove Proposition 4.3 in Section A.4. 
Many of the constructions and properties we describe here are standard and can be found, for example, in $[1,15]$. We highlight those aspects of the development that are novel as they arise.

\section{A.1 Clifford algebras}

Definition The Clifford algebra $\mathrm{Cl}(n)$ is the associative algebra ${ }^{4}$ (over the reals) with generators $e_{1}, e_{2}, \ldots, e_{n}$ and relations

$$
e_{i}^{2}=-\mathbf{1} \text { and } e_{i} e_{j}=-e_{j} e_{i}
$$

Here 1 denotes the multiplicative identity in the algebra.

Standard basis As a real vector space $\mathrm{Cl}(n)$ has dimension $2^{n}$. A basis for $\mathrm{Cl}(n)$ is given by all elements of the form

$$
e_{I}:=e_{i_{1}} e_{i_{2}} \cdots e_{i_{k}}
$$

where $I=\left\{i_{1}, i_{2}, \ldots, i_{k}\right\}$ is a subset of $[n]$ and $i_{1}<i_{2}<\cdots<i_{k}$. Here $e_{\emptyset}:=\mathbf{1}$ is the multiplicative identity element in $\mathrm{Cl}(n)$. Let us call $\left(e_{I}\right)_{I \subset[n]}$ the standard basis for $\mathrm{Cl}(n)$. With respect to this basis we can think of an arbitrary element $x \in \mathrm{Cl}(n)$ as

$$
x=\sum_{I \subset[n]} x_{I} e_{I}
$$

where the $x_{I} \in \mathbb{R}$. We equip $\mathrm{Cl}(n)$ with the inner product $\langle x, y\rangle=\sum_{I \subset[n]} x_{I} y_{I}$. Clearly the standard basis is orthonormal with respect to this inner product.

Left and right multiplication Any element $x \in \mathrm{Cl}(n)$ acts linearly on $\mathrm{Cl}(n)$ by left multiplication and by right multiplication. In other words, given $x \in \mathrm{Cl}(n)$ there are linear maps $\lambda_{x}, \rho_{x}: \mathrm{Cl}(n) \rightarrow \mathrm{Cl}(n)$ defined by $\lambda_{x}(y)=x y$ and $\rho_{x}(y)=y x$ for all $y \in \mathrm{Cl}(n)$.

It is clear from the relations (30) that $\lambda_{e_{i}}$ and $\rho_{e_{j}}$ act on the standard basis of $\mathrm{Cl}(n)$ by signed permutations. Specifically, if $I, J \subset[n]$, the corresponding entry of the signed permutation matrix $\left[\lambda_{e_{i}}\right]$ is

$$
\left[\lambda_{e_{i}}\right]_{I, J}= \begin{cases}(-1)^{|\{k \in I: k \leq i\}|} & \text { if } J=I \Delta\{i\} \\ 0 & \text { otherwise }\end{cases}
$$

and the $I, J$ entry of $\left[\rho_{e_{i}}\right]$ is

$$
\left[\rho_{e_{i}}\right]_{I, J}= \begin{cases}(-1)^{|\{k \in I: k \geq i\}|} & \text { if } J=I \Delta\{i\} \\ 0 & \text { otherwise }\end{cases}
$$

(where for two sets $I, J \subset[n], I \Delta J=(I \backslash J) \cup(J \backslash I)$ is their symmetric difference). Hence both $\lambda_{e_{i}}$ and $\rho_{e_{i}}$ are skew-symmetric. Concise descriptions of these matrices that are particularly useful for implementation are

$$
\begin{aligned}
& {\left[\lambda_{e_{i}}\right]=\overbrace{\left[\begin{array}{cc}
1 & 0 \\
0 & -1
\end{array}\right] \otimes \cdots \otimes\left[\begin{array}{cc}
1 & 0 \\
0 & -1
\end{array}\right]}^{i-1} \otimes\left[\begin{array}{cc}
0 & -1 \\
1 & 0
\end{array}\right] \otimes \overbrace{\left[\begin{array}{cc}
1 & 0 \\
0 & 1
\end{array}\right] \otimes \cdots \otimes\left[\begin{array}{cc}
1 & 0 \\
0 & 1
\end{array}\right]}^{n-i} \text { and }} \\
& {\left[\rho_{e_{i}}\right]=\underbrace{\left[\begin{array}{cc}
1 & 0 \\
0 & 1
\end{array}\right] \otimes \cdots \otimes\left[\begin{array}{cc}
1 & 0 \\
0 & 1
\end{array}\right]}_{i-1} \otimes\left[\begin{array}{cc}
0 & -1 \\
1 & 0
\end{array}\right] \otimes \underbrace{\left[\begin{array}{cc}
1 & 0 \\
0 & -1
\end{array}\right] \otimes \cdots \otimes\left[\begin{array}{cc}
1 & 0 \\
0 & -1
\end{array}\right]}_{n-i}}
\end{aligned}
$$

\footnotetext{
${ }^{4}$ That such an algebra exists and is unique up to isomorphism follows because it can be realized as a quotient of the tensor algebra.
} 
Conjugation Observe that since $\lambda_{e_{i}}^{*}=-\lambda_{e_{i}}=\lambda_{-e_{i}}$ the adjoint of left multiplication by $e_{i}$ is left multiplication by $-e_{i}$. Similarly the adjoint of right multiplication by $e_{i}$ is right multiplication by $-e_{i}$. In fact, it is the case that for any $x \in \mathrm{Cl}(n)$ there is $\bar{x} \in \mathrm{Cl}(n)$ such that $\lambda_{x}^{*}=\lambda_{\bar{x}}$ and $\rho_{x}^{*}=\rho_{\bar{x}}$. To see this define a conjugation map $x \mapsto \bar{x}$ on the standard basis by

$$
\overline{e_{I}}=(-1)^{|I|} e_{i_{k}} \cdots e_{i_{2}} e_{i_{1}}
$$

and extend by linearity. It is easy to see by direct computation that $\lambda_{e_{I}}^{*}=\lambda_{\overline{e_{I}}}$ and $\rho_{e_{I}}^{*}=\rho_{\overline{e_{I}}}$ as required. We use this conjugation map repeatedly in the sequel, usually via the relations

$$
\langle x y, z\rangle=\left\langle\lambda_{x} y, z\right\rangle=\left\langle y, \lambda_{x}^{*} z\right\rangle=\left\langle y, \lambda_{\bar{x}} z\right\rangle=\langle y, \bar{x} z\rangle
$$

and

$$
\langle y x, z\rangle=\left\langle\rho_{x} y, z\right\rangle=\left\langle y, \rho_{x}^{*} z\right\rangle=\left\langle y, \rho_{\bar{x}} z\right\rangle=\langle y, z \bar{x}\rangle
$$

Copy of $\mathbb{R}^{n}$ in $\mathbf{C l}(n)$ Throughout this appendix, we use the notation $\mathbb{R}^{n}$ to denote the $n$ dimensional subspace of $\mathrm{Cl}(n)$ spanned by the generators $e_{1}, e_{2}, \ldots, e_{n}$, and the notation $S^{n-1} \subset \mathbb{R}^{n}$ to denote the elements $x \in \mathbb{R}^{n}$ satisfying $\langle x, x\rangle=1$. We next state and prove some basic properties of the elements of $S^{n-1} \subset \mathrm{Cl}(n)$.

Lemma A.1. If $u \in S^{n-1} \subset \mathrm{Cl}(n)$ then $u \bar{u}=1$. Consequently $\langle u y, u z\rangle=\langle y, x\rangle=\langle y u, z u\rangle$ for all $y, z \in \mathrm{Cl}(n)$.

Proof. The second statement follows from the first together with (31) and (32). That $u \bar{u}=\mathbf{1}$ whenever $u \in S^{n-1}$ follows from a direct computation using the defining relations of $\mathrm{Cl}(n)$ from $(30)$.

The following can be established by repeatedly applying Lemma A.1.

Corollary A.2. If $u_{1}, u_{2}, \ldots, u_{k} \in S^{n-1}$ then $\left\langle u_{1} u_{2} \cdots u_{k}, u_{1} u_{2} \cdots u_{k}\right\rangle=1$.

Even subalgebra Consider the subspaces $\mathrm{Cl}^{0}(n)$ and $\mathrm{Cl}^{1}(n)$ of $\mathrm{Cl}(n)$ defined by

$$
\mathrm{Cl}^{0}(n)=\operatorname{span}\left\{e_{I}: I \subset[n],|I| \text { even }\right\} \quad \text { and } \operatorname{Cl}^{1}(n)=\operatorname{span}\left\{e_{I}: I \subset[n],|I| \text { odd }\right\} .
$$

It is straightforward to show that if $x, y \in \mathrm{Cl}^{0}(n)$ then $x y \in \mathrm{Cl}^{0}(n)$, and if $x, y \in \mathrm{Cl}^{1}(n)$ then $x y \in \mathrm{Cl}^{0}(n)$. The first of these properties states that $\mathrm{Cl}^{0}(n)$ is a subalgebra of $\mathrm{Cl}(n)$, which we call the even subalgebra. With these properties we have that the product of an even number of elements of $S^{n-1}$ is in the even subalgebra.

Lemma A.3. If $u_{1}, u_{2}, \ldots, u_{2 k} \in S^{n-1}$ then $x=u_{1} u_{2} \cdots u_{2 k} \in \mathrm{Cl}^{0}(n)$.

Proof. Since $S^{n-1} \subset \mathbb{R}^{n} \subset \mathrm{Cl}^{1}(n)$, each $u_{i} \in \mathrm{Cl}^{1}(n)$. Hence $u_{2 i-1} u_{2 i} \in \mathrm{Cl}^{0}(n)$ for $i=1,2, \ldots, k$. So $u_{1} u_{2} \cdots u_{2 k}=\left(u_{1} u_{2}\right)\left(u_{3} u_{4}\right) \cdots\left(u_{2 k-1} u_{2 k}\right)$ is the product of elements in $\mathrm{Cl}^{0}(n)$ so is itself an element of $\mathrm{Cl}^{0}(n)$.

The final property of elements of $\mathbb{R}^{n} \subset \mathrm{Cl}(n)$ we use in the sequel is the coordinate-free version of the defining relations of $\mathrm{Cl}(n)$ given in $(30)$.

Lemma A.4. If $u, v \in \mathbb{R}^{n}$ then

$$
u v+v u=-2\langle u, v\rangle \mathbf{1}
$$

Proof. First note that (33) is bilinear in $u$ and $v$ so it suffices to verify the identity for $u=e_{i}$ and $v=e_{j}$ (for all $\left.1 \leq i, j \leq n\right)$. That the statement holds for $u=e_{i}$ and $v=e_{j}($ for all $1 \leq i, j \leq n)$ is equivalent to the relations (30) (since $\left\langle e_{i}, e_{j}\right\rangle=\delta_{i j}$ ). 


\section{A.2 The quadratic mapping}

We now define and establish the relevant properties of the quadratic mapping $Q: \mathrm{Cl}^{0}(n) \rightarrow \mathbb{R}^{n \times n}$ that plays a prominent role in Section 4.2. Our aim is to prove Proposition 4.1. First define $\widetilde{Q}: \mathrm{Cl}(n) \rightarrow \mathbb{R}^{n \times n}$ by

$$
\widetilde{Q}(x)(u)=\pi_{\mathbb{R}^{n}} \lambda_{x} \rho_{\bar{x}}(u)=\pi_{\mathbb{R}^{n}}(x u \bar{x}) .
$$

Note that $\widetilde{Q}(x)$ is quadratic in $x$. When we express the linear map $\widetilde{Q}(x)$ as a matrix (with respect to the standard basis) we see that

$$
[\widetilde{Q}(x)]_{i j}=\left\langle e_{i}, x e_{j} \bar{x}\right\rangle .
$$

Then define $Q: \mathrm{Cl}^{0}(n) \rightarrow \mathbb{R}^{n \times n}$ as the restriction of $\widetilde{Q}$ to the subalgebra $\mathrm{Cl}^{0}(n)$.

This construction is motivated by the fact that if $u \in S^{n-1}$ then $-\widetilde{Q}(u)$ is the reflection in the hyperplane orthogonal to $u$.

Lemma A.5. Let $u \in S^{n-1}$. Then whenever $v \in \mathbb{R}^{n},-u v \bar{u} \in \mathbb{R}^{n}$ is the reflection of $v$ in the hyperplane normal to $u$. In particular $-u v \bar{u} \in \mathbb{R}^{n}$.

Proof. Let $u \in S^{n-1}$. Then by (33), if $v \in \mathbb{R}^{n}$ then $-u v=2\langle u, v\rangle \mathbf{1}+v u$ and so since $u \bar{u}=\mathbf{1}$ and $\bar{u}=-u$ it follows that

$$
-u v \bar{u}=2\langle u, v\rangle \bar{u}+v u \bar{u}=v-2\langle u, v\rangle u
$$

which is precisely the reflection in the hyperplane orthogonal to $u$ and is certainly in $\mathbb{R}^{n}$.

Note that our definition of $\widetilde{Q}$ is one possible extension to all of $\mathrm{Cl}(n)$ of the map that sends $u \in S^{n-1}$ to the reflection in the hyperplane orthogonal to $u$. It is specifically chosen so as to be quadratic on all of $\mathrm{Cl}(n)$. Our choice is different from the typical extension used in the literaturethe twisted adjoint representation [1] — which is not quadratic in $x$ on all of $\mathrm{Cl}(n)$ and is not suitable for our purposes.

Lemma A.6. Let $x \in \mathrm{Cl}(n)$ and $u \in S^{n-1}$. Then

$$
\widetilde{Q}(x u)=\widetilde{Q}(x) \widetilde{Q}(u) \quad \text { and } \quad \widetilde{Q}(u x)=\widetilde{Q}(u) \widetilde{Q}(x)
$$

where the product on the right hand side of each equality is composition of linear maps.

Proof. If $u \in S^{n-1}$, we know from the previous lemma that $v \mapsto u v \bar{u}$ leaves the subspace $\mathbb{R}^{n}$ (and hence its orthogonal complement) invariant. So by the definition of $\widetilde{Q}$ we see that

$$
\widetilde{Q}(x u)(v)=\pi_{\mathbb{R}^{n}}(x u v \bar{u} \bar{x})=\pi_{\mathbb{R}^{n}}\left(x \pi_{\mathbb{R}^{n}}^{*} \pi_{\mathbb{R}^{n}}(u v \bar{u}) \bar{x}\right)=\widetilde{Q}(x)(\widetilde{Q}(u)(v)) .
$$

Similarly since $\pi_{\mathbb{R}^{n}}^{*} \pi_{\mathbb{R}^{n}}+\pi_{\mathbb{R}^{n \perp}}^{*} \pi_{\mathbb{R}^{n \perp}}=I$,

$$
\widetilde{Q}(u x)(v)=\pi_{\mathbb{R}^{n}}(u x v \bar{x} \bar{u})=\pi_{\mathbb{R}^{n}}\left(u \pi_{\mathbb{R}^{n}}^{*} \pi_{\mathbb{R}^{n}}(x v \bar{x}) \bar{u}\right)+\pi_{\mathbb{R}^{n}}\left(u \pi_{\mathbb{R}^{n \perp}}^{*} \pi_{\mathbb{R}^{n \perp}}(x v \bar{x}) \bar{u}\right)=Q(u)(Q(x)(v))+0
$$

where we have used the fact that $u y \bar{u} \in \mathbb{R}^{n \perp}$ whenever $y \in \mathbb{R}^{n \perp}$. 


\section{A.3 $\operatorname{Spin}(n)$ and the proofs of Propositions 4.1, 4.2 and 4.4}

Definition A.7. Define $\operatorname{Spin}(n)$ as the set of all even length products of elements of $S^{n-1}$ :

$\operatorname{Spin}(n)=\left\{x \in \operatorname{Cl}(n): x=u_{1} u_{2} \cdots u_{2 k}\right.$ for some positive integer $k$ and $\left.u_{1}, \ldots, u_{2 k} \in S^{n-1}\right\}$.

Although we do not require this fact, it can be shown that in the above definition it is enough to take $k=\lfloor n / 2\rfloor$. We note that a common alternative definition [1] is to take $\operatorname{Spin}(n)$ to be the elements of $\mathrm{Cl}^{0}(n)$ satisfying $x \bar{x}=\mathbf{1}$ and $x v \bar{x} \in \mathbb{R}^{n}$ for every $v \in \mathbb{R}^{n}$ (which defines a real algebraic variety specified by the vanishing of a collection of quadratic equations). It is fairly straightforward to establish that these two definitions are equivalent.

The next result establishes that $\operatorname{Spin}(n)$ is a group under multiplication.

Lemma A.8. If $x \in \operatorname{Spin}(n)$ then $\bar{x} x=x \bar{x}=1$. If $x, y \in \operatorname{Spin}(n)$ then $x y \in \operatorname{Spin}(n)$.

Proof. That $\operatorname{Spin}(n)$ is closed under multiplication is clear from the definition. That conjugation and inversion coincide on $\operatorname{Spin}(n)$ follows from Lemma A.1.

The following result establishes Proposition 4.1 and Proposition 4.2.

Lemma A.9. $\operatorname{Spin}(n)$ is a subset of the unit sphere in $\mathrm{Cl}^{0}(n)$, i.e. $\operatorname{Spin}(n) \subset\left\{x \in \mathrm{Cl}^{0}(n)\right.$ : $\langle x, x\rangle=1\}$, satisfying $Q(\operatorname{Spin}(n))=S O(n)$. Furthermore whenever $I \subset[n]$ has even cardinality, $e_{I} \in \operatorname{Spin}(n)$.

Proof. That $\operatorname{Spin}(n) \subset\left\{x \in \mathrm{Cl}^{0}(n):\langle x, x\rangle=1\right\}$ follows directly from Lemma A.3 and Corollary A.2. Let $X \in S O(n)$. By the Cartan-Dieudonné theorem [10] any such $X$ can be expressed as the composition of an even number (at most $n$ ) of reflections in hyperplanes with normal vectors, say, $u_{1}, u_{2}, \ldots, u_{2 k} \in S^{n-1}$. Let $x=u_{1} u_{2} \cdots u_{2 k-1} u_{2 k} \in \operatorname{Spin}(n)$. Then by Lemma A.5 and Lemma A.6 and the fact that $Q$ is the restriction of $\widetilde{Q}$ to $\mathrm{Cl}^{0}(n)$,

$$
X=\widetilde{Q}\left(u_{1}\right) \widetilde{Q}\left(u_{2}\right) \cdots \widetilde{Q}\left(u_{2 k-1}\right) \widetilde{Q}\left(u_{2 k}\right)=\widetilde{Q}(x)=Q(x) \in Q(\operatorname{Spin}(n)) .
$$

Hence $S O(n) \subseteq Q(\operatorname{Spin}(n))$. On the other hand, if $x=u_{1} u_{2} \cdots u_{2 k-1} u_{2 k} \in \operatorname{Spin}(n)$ then $Q(x)$ is the product of an even number of reflections in hyperplanes and so is an element of $S O(n)$, establishing the reverse inclusion.

For the last statement, let $I=\left\{i_{1}, \ldots, i_{2 k}\right\}$ be a subset of $[n]$ with even cardinality and suppose $i_{1}<i_{2}<\cdots<i_{2 k}$. Then $e_{I}=e_{i_{1}} e_{i_{2}} \cdots e_{i_{2 k}}$ realizes $e_{I}$ as the product of an even number of elements of $S^{n-1}$, showing that $e_{I} \in \operatorname{Spin}(n)$.

We conclude the section by establishing Proposition 4.4.

Lemma A.10. If $U, V \in S O(n)$ then there is a corresponding invertible linear map $\Phi_{(U, V)}$ : $\mathrm{Cl}^{0}(n) \rightarrow \mathrm{Cl}^{0}(n)$ such that for any $x \in \mathrm{Cl}^{0}(n), U Q(x) V^{T}=Q\left(\Phi_{(U, V)} x\right)$ and $\Phi_{(U, V)}(\operatorname{Spin}(n))=$ $\operatorname{Spin}(n)$.

Proof. By Lemma A.9 there are $u, v \in \operatorname{Spin}(n)$ such that $Q(u)=U$ and $Q(v)=V$. Define $\Phi_{(U, V)}$ : $\mathrm{Cl}^{0}(n) \rightarrow \mathrm{Cl}^{0}(n)$ by $\Phi_{(U, V)}(x)=u x \bar{v}$. Then $\Phi_{(U, V)}$ is invertible with inverse $\Phi_{(U, V)}^{-1}(x)=\bar{u} x v$. Since $Q(1)=I$, by Lemma A.6 we have that whenever $v \in \operatorname{Spin}(n), Q(v)$ is orthogonal and so

$$
I=Q(1)=Q(v \bar{v})=Q(v) Q(\bar{v})=Q(v) Q(v)^{T} .
$$

Again by Lemma A.6, for any $x \in \mathrm{Cl}^{0}(n)$,

$$
U Q(x) V^{T}=Q(u) Q(x) Q(v)^{T}=Q(u) Q(x) Q(\bar{v})=Q(u x \bar{v}) .
$$

Finally, if $x \in \operatorname{Spin}(n)$ then $\Phi_{(U, V)}(x)=u x \bar{v} \in \operatorname{Spin}(n)$ by Lemma A.8. Hence $\Phi_{(U, V)}(\operatorname{Spin}(n))=$ $\operatorname{Spin}(n)$. 


\section{A.4 Matrices of the quadratic mapping (proof of Proposition 4.3)}

For $1 \leq i, j \leq n$, let $A_{i j}$ be the $2^{n-1} \times 2^{n-1}$ symmetric matrix representing the quadratic form $[Q(x)]_{i j}$, i.e.

$$
[Q(x)]_{i j}=\left\langle e_{i}, x e_{j} \bar{x}\right\rangle=\sum_{I, J \in \mathcal{I}_{\text {even }}} x_{I} x_{J}\left[A_{i j}\right]_{I, J}
$$

where $\mathcal{I}_{\text {even }}$ is the set of subsets of $[n]$ of even cardinality. We now turn to describing these matrices concretely, giving a proof of Proposition 4.3.

Proof of Proposition 4.3. We first define matrices $\widetilde{A}_{i j}$ for $1 \leq i, j \leq n$. These are the $2^{n} \times 2^{n}$ symmetric matrices representing the quadratic forms $[\widetilde{Q}(x)]_{i j}$, i.e.

$$
[\widetilde{Q}(x)]_{i j}=\left\langle e_{i}, x e_{j} \bar{x}\right\rangle=\sum_{I, J \subseteq[n]} x_{I} x_{J}\left[\widetilde{A}_{i j}\right]_{I, J} .
$$

Since

$$
\left\langle e_{i}, x e_{j} \bar{x}\right\rangle=\left\langle e_{i} x, x e_{j}\right\rangle=\left\langle x, \lambda_{\overline{e_{i}}} \rho_{e_{j}} x\right\rangle=-\left\langle x, \lambda_{e_{i}} \rho_{e_{j}} x\right\rangle
$$

it follows that

$$
\widetilde{A}_{i j}=-\lambda_{e_{i}} \rho_{e_{j}}
$$

Recall that in Section A.1 we give concrete expressions for the matrices defining $\lambda_{e_{i}}$ and $\rho_{e_{j}}$. Hence (34) gives a convenient way to explicitly build $\widetilde{A}_{i j}$. Note that $\lambda_{e_{i}} \rho_{e_{j}}$ is symmetric because $\lambda_{e_{i}}$ and $\rho_{e_{j}}$ are skew-symmetric and $\lambda_{e_{i}}$ and $\rho_{e_{j}}$ commute (because the operations of left- and right-multiplication commute in an associative algebra).

We now obtain concrete expressions for the $A_{i j}$ (rather than the $\widetilde{A}_{i j}$ ). Note that since $Q$ is the restriction of $\widetilde{Q}$ to the subspace $\mathrm{Cl}^{0}(n)$, so for each $1 \leq i, j \leq n, A_{i j}$ is the $2^{n-1} \times 2^{n-1}$ principal

submatrix of $\widetilde{A}_{i j}$ indexed by rows and columns corresponding to the basis elements $\left(e_{I}\right)_{\mathcal{I}_{\text {even }}}$ of $\mathrm{Cl}^{0}(n)$. This submatrix can be extracted by computing

$$
A_{i j}=P_{\text {even }}^{T} \widetilde{A}_{i j} P_{\text {even }}
$$

where $P_{\text {even }}$ is the $2^{n} \times 2^{n-1}$ matrix with exactly one non-zero entry per column and at most one non-zero entry per row given by

$$
P_{\text {even }}=\frac{1}{2}\left[\begin{array}{l}
I_{2^{n-1}}+\left[\begin{array}{cc}
1 & 0 \\
0 & -1
\end{array}\right] \otimes \cdots \otimes\left[\begin{array}{cc}
1 & 0 \\
0 & -1
\end{array}\right] \\
I_{2^{n-1}}-\left[\begin{array}{cc}
1 & 0 \\
0 & -1
\end{array}\right] \otimes \cdots \otimes\left[\begin{array}{cc}
1 & 0 \\
0 & -1
\end{array}\right]
\end{array}\right]
$$

which verifies (6).

\section{References}

[1] M. F. Atiyah, R. Bott, and A. Shapiro. Clifford modules. Topology, 3:3-38, 1964.

[2] A. Barvinok and G. Blekherman. Convex geometry of orbits. Combinatorial and computational geometry, 52:51-77, 2005. 
[3] A. Barvinok and A. M. Vershik. Convex hulls of orbits of representations of finite groups and combinatorial optimization. Funct. Anal. Appl., 22(3):224-225, 1988.

[4] G. Blekherman, P. A. Parrilo, and R. R. Thomas, editors. Semidefinite optimization and convex algebraic geometry, volume 13 of MPS-SIAM series on optimization. SIAM, 2013.

[5] J. Briët, D. Dadush, and S. Pokutta. On the existence of $0 / 1$ polytopes with high semidefinite extension complexity. In Algorithms-ESA 2013, pages 217-228. Springer, 2013.

[6] R. D. Carr and G. Konjevod. Polyhedral combinatorics. In Tutorials on emerging methodologies and applications in Operations Research, pages 2-1-2-48. Springer, 2005.

[7] C. B. Chua. Relating homogeneous cones and positive definite cones via T-algebras. SIAM J. Optim., 14(2):500-506, 2003.

[8] H. Fawzi, J. Saunderson, and P. A. Parrilo. Equivariant semidefinite lifts and sum-of-squares hierarchies, 2013. arXiv:1312.6662.

[9] M. Fazel. Matrix rank minimization with applications. PhD thesis, Stanford University, 2002.

[10] J. Gallier. The Cartan-Dieudonné theorem. In Geometric Methods and Applications, pages 197-247. Springer, 2001.

[11] K. Gatermann and P. A. Parrilo. Symmetry groups, semidefinite programs, and sums of squares. J. Pure Appl. Algebra, 192(1):95-128, 2004.

[12] J. Gouveia and T. Netzer. Positive polynomials and projections of spectrahedra. SIAM J. Optim., 21(3):960-976, 2011.

[13] J. Gouveia, P. A. Parrilo, and R. R. Thomas. Lifts of convex sets and cone factorizations. Math. Oper. Res., 38(2):248-264, 2013.

[14] J. Gouveia, R. Z. Robinson, and R. R. Thomas. Worst-case results for positive semidefinite rank, 2013. arXiv:1305.4600.

[15] F. R. Harvey. Spinors and Calibrations, volume 9 of Perspectives in Mathematics. Academic Press, Inc., Boston, MA, 1990.

[16] A. Horn. Doubly stochastic matrices and the diagonal of a rotation matrix. American J. Math., 76(3):620-630, 1954.

[17] M. B. Horowitz, N. Matni, and J. W. Burdick. Convex relaxations of SE(2) and SE(3) for visual pose estimation, 2014. arxiv:1401.3700.

[18] R. G. Jeroslow. On defining sets of vertices of the hypercube by linear inequalities. Discrete Math., 11(2):119-124, 1975.

[19] J. E. Keat. Analysis of least-squares attitude determination routine DOAOP. Technical Report CSC/TM-77/6034, Computer Sciences Corporation, February 1977.

[20] J. Löfberg. YALMIP: A toolbox for modeling and optimization in MATLAB. In Proc. CACSD Conf., Taipei, Taiwan, 2004.

[21] M. Longinetti, L. Sgheri, and F. Sottile. Convex hulls of orbits and orientations of a moving protein domain. Discrete Comput. Geom., 43(1):54-77, 2010. 
[22] K. V. Mardia and P. E. Jupp. Directional statistics, volume 494. John Wiley \& Sons, 2009.

[23] A. Naor, O. Regev, and T. Vidick. Efficient rounding for the noncommutative Grothendieck inequality, 2012. arXiv:1210.7656.

[24] A. Nemirovski. Sums of random symmetric matrices and quadratic optimization under orthogonality constraints. Math. Prog., 109(2-3):283-317, 2007.

[25] M. L. Psiaki. Generalized Wahba problems for spinning spacecraft attitude and rate determination. J. Astronautical Sciences, 57(1-2):73-92, 2009.

[26] M. Ramana and A. J. Goldman. Some geometric results in semidefinite programming. J. Global Optim., 7(1):33-50, 1995.

[27] J. Renegar. Hyperbolic programs, and their derivative relaxations. Found. Comput. Math., 6(1):59-79, 2006.

[28] R. T. Rockafellar. Convex analysis. Princeton University Press, 1997.

[29] R. Sanyal, F. Sottile, and B. Sturmfels. Orbitopes. Mathematika, 57(02):275-314, 2011.

[30] J. Saunderson, P. A. Parrilo, and A. S. Willsky. A semidefinite optimization approach to joint attitude and spin-rate estimation, 2014. In preparation.

[31] A. Schrijver. Combinatorial optimization: polyhedra and efficiency. Springer, 2003.

[32] R. Sinn. Algebraic boundaries of SO(2)-orbitopes. Discrete Comput. Geom., 50(1):219-235, 2013.

[33] K.-C. Toh M. J. Todd and R. H. Tütüncü. SDPT3 - a MATLAB software package for semidefinite programming, version 1.3. Optim. Methods Softw., 11(1-4):545-581, 1999.

[34] È. B. Vinberg. The theory of homogeneous convex cones. Trans. Moscow Math. Soc., 12:340403, 1965.

[35] G. Wahba. A least squares estimate of satellite attitude. SIAM Rev., 7(3):409-409, 1965. 